\title{
High Velocity Infrared Thermography and Numerical Trajectories of Solid Particles in Compressible Gas Flow
}

\author{
M. Lappa ${ }^{1}$, A. Esposito ${ }^{2}$, F. Aponte ${ }^{2}$ and C. Allouis ${ }^{3}$ \\ ${ }^{1}$ Department of Mechanical and Aerospace Engineering, University of Strathclyde, James Weir \\ Building, 75 Montrose Street, Glasgow, G1 1XJ, UK - email: marcello.lappa@strath.ac.uk \\ ${ }^{2}$ Department of Industrial Engineering, University of Naples "Federico II”, Via Claudio 21, 80125 \\ Naples, Italy \\ ${ }^{3}$ Institute of Combustion Research, National Council of Research, Via Claudio 21, Naples, Italy
}

\begin{abstract}
The use of High Velocity Infrared Thermography as a valuable alternative to other existing techniques for the visualisation and tracking of solid particles transported by a gas jet has been assessed by considering different situations in terms of problem characteristic numbers (jet Reynolds and Mach numbers and Particle Stokes and gravitational Froude numbers). Particles paths have also been calculated by means of a hybrid Eulerian-Lagrangian technique under the intent to cross-validate the two (experimental and numerical) approaches. The results indicate that such a strategy is robust and sufficiently flexible to be used in relatively wide regions of the space of parameters. Experiments have clearly demonstrated that thermography can properly capture particle dynamics with a level of detail comparable to that provided by simulations. Computations have proved to be valuable on their own by allowing the explorations of regions of the parameters space otherwise out of reach. Different tests have been conducted considering both isolated particles and "swarms". We show that the observed dynamics are induced by the delicate interplay of different effects, including inertial, gravitational and eventually "lift" contributions produced by a nonperfect horizontal orientation of the jet or other uncertainties (such as those due to a non mono-sized set of particles).
\end{abstract}

Key words: Multiphase Flow, solid particles, jet, High Velocity Infrared Thermography, hybrid Eulerian-Lagrangian approach.

\section{Introduction}

Many environmental and industrial processes involve the interaction between coherent flow structures (e.g., a jet) and particles, bubbles or droplets. These systems can generally involve a relatively wide range of characteristic length and time scales (with regard to both the flow and the motion of particles; Scala [1]; Lappa et al. [2]). Given the number and variety of relevant natural or environmental processes and potential applications in technology, providing an exhaustive description of this subject is not as straightforward as one would imagine. For these reasons (and owing to space limitations), we do not strive to review all aspects of it; rather we use the present introduction to provide the reader with a brief account of the several problems which are currently 
attracting significant interest as archetypal systems for the improvement of common technological applications.

Typical examples of industrial processes are represented by circulating fluidized beds (Yang et al. [3]), fluidized bed combustion (Scala [4]), cyclone separators (Cortés and Gil [5]), air classifiers, jet mills (Rodnianski et al. [6]) and pneumatic transport of powders (Molerus [7]). Other technological cases relating to this subject include spray combustion devices and fuel injectors (Thimothée et al. [8]; Kuo [9]; Aleiferis and van Romunde [10]), spray coating and painting (Heimann [11]; Pasandideh-Fard et al. [12]), laser energy deposition techniques (Ukai et al. [13]), pulverized coal furnaces (Chen et al. [14]) and solid propellant rockets (Lomba et al. [15]; Najjar [16]). Last but not least, also convectively unstable fluids transporting fine dispersed particles should be considered as instances pertaining to this subject (Lappa [17]), e.g., rising plumes originating from urban mass fires, the release in the atmosphere of smokes from industrial stacks (McGrattan et al. [18]), severe accidents in chemical or nuclear reactors (Kissane [19]), and pyroclastic flows from volcanic eruptions (Valentine and Wohletz [20]; Haszpra and Tél [21]). Other examples with relevance to the environment include droplet dispersion in agricultural sprays (De Cock et al. [22]) and the transport of sediment in rivers and coastal regions (Yang [23]).

Given the wide-ranging nature of all these subjects, the reader should not be surprised by the fact that the general problem connected with the interactions of particles with a coherent flow has been approached by different investigators with different backgrounds and perspectives. In general, the description of such multiphase systems poses fundamental theoretical questions as well as difficulties in terms of theoretical and mathematical modelling. Also the experimental approach has often to deal with complex issues which can make the analysis ill-posed.

To put our work in perspective (transcending for a while potential applications), in the remainder of this introduction we provide a brief summary of the relevant "fundamental" studies in the literature from which we took inspiration. Along these lines we start from the work by Perkins et al. [24] where a series of experiments were conducted with dense particles released above a horizontal plane turbulent air jet and collected in trays beneath the jet. These experiments were simulated using a Random-Flight model, and the predicted distributions compared with those measured in the experiments. Good agreement was obtained for the large particles, but the agreement was very poor for the smaller particles which seemed to leave the jet much sooner than the model predicted.

In such a context, it is also worth mentioning the experiments conducted by Kennedy and Moody [25] about monodisperse hexadecane droplets injected onto the centreline of a turbulent air jet. These authors measured particle dispersion and particle velocities with laser sheets and a position sensitive photomultiplier tube (their radial dispersion, axial velocities, and times of flight were measured as functions of axial position). The time and length scales of the jet were varied through the control of the jet exit velocity and nozzle diameter. Wide ranges of the Reynolds numbers and particle Stokes numbers were covered. The times-of-flight were used to analyse the dispersion measurements in terms of Lagrangian statistics. 
More recently, the problem has been approached by means of phase Doppler anemometry. As an example, Decker et al. [26] measured in this way the radial profiles of particle mean velocities, particle sizes, rms velocities and turbulence intensities. Such experiments were executed in a jet at different axial and radial distances from the nozzle. The variation of the initial velocity conditions, particle diameter distributions, and particle loadings were found to provide meaningful information about the local flow structures and their effect on the macroscopic as well as the turbulent particle transport between the jet centre and the outer shear layer.

Towards the end to develop an alternative point of view on these subjects, in the present work, we implement an approach based on the application of HVIT (High Velocity Infrared Thermography). Of course, in the past this technique has been already used in various companion fields, e.g., for fluidized beds (where solid particles in the beds are fluidized by injected gas flow and the gas goes through the small gaps between the particles, Tsuji et al. [27]), or to track the motion of particles at the interface between two fluids (based on the employment of highly reflective particles which, when suspended near or at the interface, can be distinguished from the surrounding fluid domain due to their different emissivity). Examples along these lines are the works by Charogiannis et al. [28], who used this strategy to study gravity-driven liquid-film flows over inclined heated substrates, and Segura et al. [29], who applied a similar method in the context of typical microfluidics applications. Using a slightly different approach, Someya et al. [30] used temperature-sensitive fluorescent particles, i.e. particles, treated with a temperature dependent luminophore in experiments dealing with thermal convection.

Despite these valuable efforts, the application of thermography to high-velocity (compressible flow) fluid-particle systems, however, seems to be still relatively limited. For such cases, indeed, other traditional techniques such as Doppler anemometry or multiframe laser shadow visualization methods are preferred (see, e.g., Boiko et al. [31]). To the best of our knowledge, only Fond et al. [32] and Abram et al. [33] applied optical diagnostic technique based on seeded thermographic phosphor particles to compressible gas flow (turbulent jets typically occurring in combustors). Their method relies on a remarkable physical property of matter, namely, that as a result of simultaneous UV excitation, these particles can emit phosphorescence with a temperature-dependent emission spectrum (when two spectrally filtered images of the particle phosphorescence emission are recorded, the tracer temperature can be determined using a ratio-based method). The focus of these experimental investigation, however, was not particle dynamics; rather particles were used to determine gas velocity assuming that, since their size is very small, they rapidly assume the temperature and velocity of the surrounding gas.

In the present short work, thermography is specifically used to track particle trajectory. In addition, dedicated numerical simulations relying on a hybrid Eulerian-Lagrangian approach are considered with the two-fold aim to cross-validate the two (experimental and numerical) approaches and extend the study to regions of the space of parameters not explorable with the available hardware (experimental facilities). 


\section{Mathematical and Numerical Model}

A proper treatment of multi-phase phenomena involving the transport of solid particles in a jet of gas requires the problem to be modelled in a consistent way at different scales simultaneously. The first of these scales is represented by the size of the physical domain or the extension of the considered gas jet. The second scale in such a modelling hierarchy obviously relates to the involved particles and their motion. In turn, mathematical modelling of the coupling between these processes has to rely on adequate representation of the interaction occurring between the particles and the surrounding gas.

\section{Nomenclature}

D Jet diameter [m]

E total energy per unit mass $\left[\mathrm{JKg}^{-1}\right]$

F inviscid flux (array)

Fr Froude number [-]

g Gravity acceleration $\left[\mathrm{ms}^{-2}\right]$

$\mathrm{G}$ inviscid flux (array)

M Mach number [-]

p Pressure $\left[\mathrm{Nm}^{-2}\right]$

q Array containing the problem unknowns

$\mathrm{R} \quad$ Radius [m] or gas constant $\left[\mathrm{JKg}^{-1} \mathrm{~K}^{-1}\right]$

Re Reynolds number [-]

St Stokes number [-]

$\mathrm{T}$ Temperature [K]

$\mathrm{t} \quad$ Time $[\mathrm{s}]$

$\mathrm{u} \quad$ Horizontal velocity component $\left[\mathrm{ms}^{-1}\right]$

$V \quad$ Velocity $\left[\mathrm{ms}^{-1}\right]$

$\mathrm{v} \quad$ Vertical velocity component $\left[\mathrm{ms}^{-1}\right]$

$\mathrm{x} \quad$ Horizontal Cartesian coordinate [m]

y Vertical Cartesian coordinate [m]

Subscripts

flow flow

g gas

JET jet

o reference condition

p particle

part particle 
$\begin{array}{ll}\text { sett } & \text { settling } \\ \text { suth } & \text { Sutherland } \\ \mathrm{T} & \text { terminal }\end{array}$

Greek and other symbols

$\alpha \quad$ thermal diffusivity $\left[\mathrm{m}^{2} \mathrm{~s}^{-1}\right]$

$\gamma \quad$ ratio of gas specific heats [-]

$\mathfrak{R} \quad$ non-dimensional ratio of particle sedimentation velocity and jet velocity [-]

$v \quad$ kinematic viscosity $\left[\mathrm{m}^{2} \mathrm{~s}^{-1}\right]$

$\theta \quad$ inclination angle [degrees]

$\mu \quad$ dynamic viscosity $\left[\mathrm{Nsm}^{-1}\right]$

$\xi \quad$ density ratio [-]

$\rho$ density $\left[\mathrm{kgm}^{-3}\right]$

$\tau \quad$ characteristic time $[\mathrm{s}]$

\subsection{The Fluid Phase}

To represent the gas phase we use a standard Eulerian description, that is, the gas characteristics are calculated at fixed positions (grid points) in space. As the flow that we consider is compressible (or weakly compressible) and the related Reynolds number $\left(\operatorname{Re}_{J E T}=V_{J E T} \mathrm{D} / v\right.$ where $\mathrm{V}_{\mathrm{JET}}$ is a characteristic velocity for the considered jet, $\mathrm{D}$ its diameter and $v$ a representative reference value for the gas kinematic viscosity) is relatively high $\left(\geq O\left(10^{4}\right)\right)$, we rely on the inviscid twodimensional Euler equations. These equations can be cast in condensed (matrix) form as

$\frac{\partial \underline{q}}{\partial t}+\frac{\partial \underline{F}}{\partial x}+\frac{\partial \underline{G}}{\partial y}=0$

where the vector quantity $q$ is an array containing the problem unknowns, i.e. density, momentum and energy:

$q=\left[\rho_{g}, \rho_{g} u, \rho_{g} v, \rho_{g} E\right]$

$\rho_{g}$ being the density of the gas, $u$ and $v$ its velocity components along the $x$ and $y$ directions, respectively, and $E$ the total energy per unit mass. The related inviscid fluxes, in turn, can be expressed as: 
$\underline{F}=\left[\begin{array}{c}\rho_{g} u \\ \rho_{g} u^{2}+p \\ \rho_{g} u v \\ \rho_{g} u\left(E+\frac{p}{\rho_{g}}\right)\end{array}\right] \quad \underline{G}=\left[\begin{array}{c}\rho_{g} v \\ \rho_{g} v u \\ \rho_{g} v^{2}+p \\ \rho_{g} v\left(E+\frac{p}{\rho_{g}}\right)\end{array}\right]$

where $E=C_{v} T+\frac{1}{2}\left(u^{2}+v^{2}\right)$ and $p=\rho_{g} R T$ (the gas state equation, $R$ being the gas constant and $\mathrm{C}_{\mathrm{v}}$ is the specific heat coefficient; as reference conditions we assume air at atmospheric pressure and $288 \mathrm{~K} \rightarrow v_{0}=1.46 \times 10^{-5} \mathrm{~m}^{2} / \mathrm{s}$ and $\rho_{0}=1.23 \mathrm{Kg} / \mathrm{m}^{3}$ ).

By using the inviscid Euler equations we also implicitly assume that the heat diffusive exchange (conduction) between the jet and the external environment is negligible. This assumption is indeed supported by the very high value taken by the ratio of the jet thermal diffusion time, defined as $\mathrm{D}^{2} / \alpha$ where $\alpha$ is the gas thermal diffusivity, and the convective transport time, defined as $\mathrm{D} / V_{J E T}$. Such ratio reads $V_{J E T} \mathrm{D} / \alpha=\operatorname{Pr} \operatorname{Re}_{\mathrm{JET}}$. As for the conditions considered in the present work, $\operatorname{Re}_{\mathrm{JET}}>>1$, and the value taken by the Prandtl number $\operatorname{Pr}=v / \alpha$ is $\cong 1$, the condition $\operatorname{PrRe} \operatorname{RET}_{\text {JET }}>>1$ implies that the impact of diffusive heat exchange on the temperature of the jet can be considered negligible for all the conditions considered in the present work .

\section{$\underline{2.2 \text { Particle Tracking }}$}

As anticipated, two approaches running in parallel have to be implemented to address adequately the present problem, one targeting the large-scale flow, and the other able to capture small-scale effects, namely particle motion and related mechanisms. The models existing in the literature in this regard can generally be split into two main categories, namely a class entirely based on the assumption of "continuum" (where particles are assumed to behave as a "fluid", which can mix with the carrier fluid) or partially involving a discrete representation of reality (with particles behaving as independent entities tracked separately with respect to the background fluid flow).

Each approach, of course, has its own merits and disadvantages. The first (see, e.g., Marble [34]; Dasgupta et al. [35]; De’ Michieli Vitturi et al. [36]), which may be regarded essentially as an Eulerian description of the dispersed phase, works particularly well for the case of very dense dispersions of particles in gases (in such cases, typically, the gas-particle mixture is treated as a homogeneous medium through the adoption of an "equivalent gas model”).

As in the present work, however, we concentrate on a single or a limited number of particles, an approach such as that described above (based on the Eulerian description) is not applicable. This is the main reason for which we rely on the second category of models mentioned above (namely a Lagrangian representation where each particle is tracked as a separate entity interacting with the gas). 
Before providing the reader with the details about the specific equation used to track the particle, in the following, however, first we lay the general foundations of our theoretical treatment of the dispersed phase in terms of relevant effects and related characteristic numbers.

Along these lines, we start from the simple remark that in the present case, the density $\rho_{p}$ of the particles is much larger than the density $\rho_{g}$ of the surrounding gas ( $\xi=\rho_{p} / \rho_{g}>1$, which implies that the inertia of the former cannot be neglected). Moreover, the particles have a characteristic size very small with respect to the length scale of the considered physical domain, which leads to the need to account for viscous effects at the particle length scale (Lappa et al. [2]).

On the one hand, such size is so small that viscous effects cannot be neglected, on the other hand, however, it is not infinitesimal, which makes a representation based on the concepts of point-like particles with negligible mass too inaccurate (as explained above, a physically accurate model must take into account the particle inertia). A first relevant quantity leading to cast the problem in a relevant theoretical framework is the so-called particle relaxation (or aerodynamic response) time $(\tau)$, which in the literature ([2]) is generally defined as:

$$
\tau_{\text {part }}=\frac{2}{9} \frac{R_{p}^{2}}{v_{0}}
$$

As the characteristic time scale of the fluid flow, without loss of generality, it is possible to assume:

$$
\tau_{\text {flow }}=\frac{D^{2}}{v_{0}}
$$

Where $R_{p}$ is the particle radius and $v_{0}$ is the reference fluid kinematic viscosity.

Moreover, to take into account the effect of the particle "weight", we introduce a third relevant time scale, namely, the particle setting time:

$$
\tau_{\text {sett }}=\frac{9}{2} \frac{v_{0} D}{g R_{p}^{2}}
$$

which relates to the time that a particle falling with its terminal velocity would require to move along a distance equal to the reference length $\mathrm{D}$ (the jet diameter).

While this scale can be seen as a time scale accounting for the forcing of steady nature that a particle undergoes due to the Earth's gravitational field (this being linked, in turn, to gravity acceleration, particle size and fluid viscosity only), $\tau_{\text {flow }}$ can be seen as a measure of the time needed by the particle to react to unsteady forcing eventually produced by its interaction with the carrier flow.

Given such premises, specific additional nondimensional parameters can be introduced as the ratio of the characteristic times so far introduced. Such nondimensional numbers read: 


$$
\frac{\tau_{\text {part }}}{\tau_{\text {flow }}}=\frac{2}{9} \frac{R_{p}^{2}}{D^{2}}=S t
$$

generally known as the particle Stokes number, and:

$$
\begin{aligned}
& \frac{\tau_{\text {sett }}}{\tau_{\text {part }}}=\left(\frac{9}{2}\right)^{2} \frac{D v_{0}^{2}}{g R_{p}^{4}}=\frac{F r^{2}}{S t^{2}} \\
& \frac{\tau_{\text {sett }}}{\tau_{\text {flow }}}=\left(\frac{9}{2}\right) \frac{v_{0}^{2}}{g D R_{p}^{2}}=\frac{F r^{2}}{S t}
\end{aligned}
$$

which, as shown by (Raju and Meiburg [37]) can be also written in combined form as :

$$
\sqrt{\frac{\tau_{\text {sett }}}{\tau_{\text {flow }}} \frac{\tau_{\text {part }}}{\tau_{\text {flow }}}}=\frac{v_{0}}{D^{3 / 2} \sqrt{g}}=F r
$$

As the reader will easily realise at this stage, the condition St $\rightarrow 0$ corresponds to the situation for which the particle would be seen (by a fixed observer) moving with the same velocity as the carrier flow, i.e. particles "frozen" in the carrier flow. By contrast, the opposite limit St $>>1$, represents circumstances for which, owing to excessive inertia, particles would not be able to respond to any velocity change occurring in the external gas.

Similarly, the other two nondimensional ratios can be used to characterize the "residence time" of the particle inside the jet, i.e. the timeframe in which the carrier flow can interact with a particle by delaying its settling. For a fixed jet diameter D, the condition $\mathrm{Fr} \rightarrow 0$ would therefore correspond to the case of a particle undergoing undisturbed purely vertical displacement with its terminal velocity:

$$
V_{T}=\frac{2}{9} R_{p}^{2} \frac{g(\xi-1)}{v}
$$

Another very useful parameter in this regard can be introduced as the non-dimensional ratio between the ideal particle sedimentation velocity $V_{T}$ defined above (which would be attained by a particle in a purely quiescent medium) and the jet velocity $V_{J E T}$. This parameter can formally be expressed as:

$$
\mathfrak{R}=\frac{V_{T}}{V_{J E T}}=(\xi-1) \frac{S t}{\operatorname{Re}_{J E T} F r^{2}}
$$


which indicates that the relative importance of these two characteristic velocities is directly proportional to the Stokes number and inversely proportional to the jet Reynolds number and the square of the Froude number (implying that the higher $\mathrm{Re}_{\mathrm{JET}}$ or $\mathrm{Fr}$ or the smaller $S t$, the longer will be the horizontal distance a particle can cover before hitting the bottom of the considered domain).

Given such arguments, and taking into account that the dispersed phase is constituted of small particles having fixed shape (they are undeformable), as anticipated at the beginning of this section, we resort to a "hybrid" formulation. In other words, we consider an Eulerian approach for the largescale flow (Eqs. (1) and (2)) coupled with a Lagrangian method to capture related particle dynamics. As a result, particles are treated as microscopic quantities with respect to the field variables and their unsteady motion is resolved using a force-balance equation accounting for inertial, viscous (drag) and other effects. Such an equation (see, e.g., Pérez-Munuzuri [38]) can be cast in compact vector form as

$$
\begin{aligned}
& \rho_{p} \frac{d \underline{V}}{d t}=\rho_{g} \frac{D \underline{V}}{D t}+\left(\rho_{p}-\rho_{g}\right) \underline{g}+\frac{9}{2} \frac{\mu}{R_{p}^{2}} f\left(\operatorname{Re}_{p}\right)\left(\underline{V}-\underline{V}_{p}\right)+ \\
& \frac{\rho_{g}}{2}\left(\frac{D \underline{V}}{D t}-\frac{d \underline{V}_{p}}{d t}\right)+\frac{1}{2}\left(\underline{V} \frac{D \rho_{g}}{D t}-\underline{V}_{p} \frac{d \rho_{g}}{d t}\right)
\end{aligned}
$$

where $\underline{V}_{p}=\left[u_{p}, v_{p}\right]$ is the particle velocity and $\underline{V}=[u, v]$ is the local gas velocity. Moreover, $\operatorname{Re}_{p}$ is the particle instantaneous Reynolds number, defined as

$$
\operatorname{Re}_{p}=\frac{2 R_{p} \rho_{g}\left|\underline{V}-\underline{V}_{p}\right|}{\mu} .
$$

The five terms at the right-hand side of Eq. (10) represent, respectively, the force exerted by the undisturbed flow, the buoyancy, the Stokes drag, the virtual-added mass and the additional force produced by compressibility effects (as shown by Parmar et al. [39], this last force can be expressed as it appears in eq. (10) only if the considered flow is not affected by the presence of shock waves). The additional term $f\left(R e_{p}\right)$ appearing in front of the drag is a corrective factor required to account for the departure of the drag from the classical Stokes law (Michaelides [40]).

$$
f\left(\operatorname{Re}_{p}\right)=1+0.15 \operatorname{Re}_{p}^{0.687}+0.0175\left(1+\frac{42500}{\operatorname{Re}_{p}^{1.16}}\right)
$$

The gas dynamic viscosity $\mu$ can be determined via the Sutherland's law on the basis of the local (at particle position) temperature:

$$
\mu_{\text {Suth }}=\mu_{o}\left(\frac{T}{T_{o}}\right)^{3 / 2} \frac{T_{o}+S}{T+S}
$$


where $\mu_{o}$ is a reference viscosity (in the same units as $\mu$ ) at a reference temperature $T_{o}$.

\section{$\underline{2.3 \text { Space discretization }}$}

Using the classical two-dimensional van Leer splitting over a uniform mesh (Hirsch [41]), the flux can be split into two components to be discretized using upwind stencils.

In the following, in particular, we implement the variant of flux splitting originally introduced by Laney [42]. In the related expressions, the Mach number for subsonic flow is given by $M^{ \pm}=$ $\pm 1 / 4(M \pm 1)^{2}$ :

$$
\underline{F}^{ \pm}= \pm \frac{\rho_{g}}{4 a}(u \pm a)^{2}\left[\begin{array}{c}
\frac{(\gamma-1) u \pm 2 a}{\gamma} \\
v \\
\frac{v^{2}}{2}+\frac{[(\gamma-1) u \pm 2 a]^{2}}{2\left(\gamma^{2}-1\right)}
\end{array}\right] \quad \underline{G}^{ \pm}= \pm \frac{\rho_{g}}{4 a}(v \pm a)^{2}\left[\begin{array}{c}
1 \\
u \\
\frac{(\gamma-1) v \pm 2 a}{\gamma} \\
\frac{u^{2}}{2}+\frac{[(\gamma-1) v \pm 2 a]^{2}}{2\left(\gamma^{2}-1\right)}
\end{array}\right]
$$

( $a$ being the local speed of sound and $\gamma$ the ratio of the specific heat coefficients).

As shown by eq. (14), the flux becomes a combination of both plus and minus flux contributions, with each component appropriately stencilled. Such an arrangement is summarized in Eq. (15):

$-1<\mathrm{M}<1 \quad \underline{F}=\underline{F}^{+}\left(\underline{q}_{\text {left }}\right)+\underline{F}^{-}\left(\underline{q}_{r i g h t t}\right)$.

\subsection{Time Integration}

By applying an explicit MacCormack scheme directly to Eq. (1), two macroscopic steps must be implemented, namely:

Predictor step

$\underline{q}_{i, j}^{*}=\underline{q}_{i, j}^{n}-\frac{\Delta t}{\Delta x}\left(\underline{F}_{i+1, j}^{n}-\underline{F}_{i, j}^{n}\right)-\frac{\Delta t}{\Delta y}\left(\underline{G}_{i, j+1}^{n}-\underline{G}_{i, j}^{n}\right)-\Delta t \underline{S}_{i, j}^{n}$

Corrector step

$\underline{q}_{i, j}^{n+1}=\frac{1}{2}\left[\underline{q}_{i, j}^{n}+\underline{q}_{i, j}^{*}-\frac{\Delta t}{\Delta x}\left(\underline{F}_{i, j}^{*}-\underline{F}_{i-1, j}^{*}\right)-\frac{\Delta t}{\Delta y}\left(\underline{G}_{i, j}^{*}-\underline{G}_{i, j-1}^{*}\right)\right]-\Delta t \underline{S}_{i, j}^{n}$ 
In this way, a method with second-order accuracy is obtained with respect to both time and space. Towards the end to enhance the stability of the algorithm and prevent it from developing spurious oscillations, we further correct this scheme with fourth-order numerical dissipation terms (Anderson [43]):

$$
\begin{gathered}
\varepsilon(|u|+a) \frac{p_{i+1, j}+p_{i-1, j}-2 p_{i, j}}{p_{i+1, j}+p_{i-1, j}+2 p_{i, j}}\left(\underline{q}_{i+1, j}-2 \underline{q}_{i, j}+\underline{q}_{i-1, j}\right), \\
\varepsilon(|v|+a) \frac{p_{i, j+1}+p_{i, j-1}-2 p_{i, j}}{p_{i, j+1}+p_{i, j-1}+2 p_{i, j}}\left(\underline{q}_{i, j+1}-2 \underline{q}_{i, j}+\underline{q}_{i, j-1}\right) .
\end{gathered}
$$

where $\varepsilon$ is a small value of $\mathrm{O}\left(10^{-2}\right)$.

We have devoted a similar care to the particle tracking equation, which has been integrated in the framework a standard fourth-order accurate explicit Runge-Kutta technique. In particular, the gas velocity $\underline{V}$ appearing in Eqs. (10) and (11) has been "reconstructed" at each particle location from the surrounding grid locations by means of a proper interpolation scheme (Lappa et al. [2]).

According to a grid refinement study, a mesh with 500 points along the vertical direction (corresponding to $18 \mathrm{~cm}$ ) has been used. The number of points along the horizontal direction has been scaled accordingly (2400 points per meter). The overall mathematical framework was validated by Lappa et al. [2]. The related details are not reported here to avoid duplications and save space. The interested reader is referred to that work for additional details. Additional evidence for the relevance of such numerical approach is provided in Sect. 4 through comparison between experiments and simulations.

\section{Experimental Facilities and related Techniques}

To track tiny particles with diameter in the range of tens of microns, a visualization system with an excellent spatial and temporal resolution has to be regarded as a mandatory pre-requisite. Also the choice of a relevant setup for jet formation and particle injection must be considered as a relevant aspect of the problem.

Such details are provided in the following sections. In particular, while Sect. 3.1 is dedicated to a short survey of the particles used for the experiments, the following two sections describe the different facilities that we have used to investigate the particle dynamics for different flow regimes (corresponding to almost incompressible hot flow, weakly compressible and compressible cold flow). The last sub-section (Sect. 3.4) is used to describe the infrared camera and the accessory setup implemented for particle injection. 


\section{$\underline{3.1 \text { Particles Characterization }}$}

Different particles have been used for the experiments (see Fig. 1). More specifically:

a) spherical sugar particles, nominal diameter $1.69 \times 10^{-3}(\mathrm{~m})$, mass $3.6 \times 10^{-6}(\mathrm{Kg})$, density 1393 $\left(\mathrm{Kg} / \mathrm{m}^{3}\right)$ and volume $2.58 \times 10^{-9}\left(\mathrm{~m}^{3)}\right.$ for single and small group particle injection in a hot carrier flow (low subsonic regime);

b) plastic spheres having $6(\mathrm{~mm})$ diameter and $0.15 \times 10^{-3}(\mathrm{Kg})$ mass, density $1326\left(\mathrm{Kg} / \mathrm{m}^{3}\right)$ for single particle injection in a cold carrier flow (high subsonic regime).

The particle diameter was measured by means of a digital calibre, while the related mass was estimated using a digital balance having a resolution of $10^{-8}(\mathrm{~kg})$, see Figure 2. Measurements of particles pertaining to group (a) revealed a non-uniform distribution of size with the diameter oscillating between $80 \%$ and $120 \%$ of the nominal value $\left(1.69 \times 10^{-3} \mathrm{~m}\right)$.

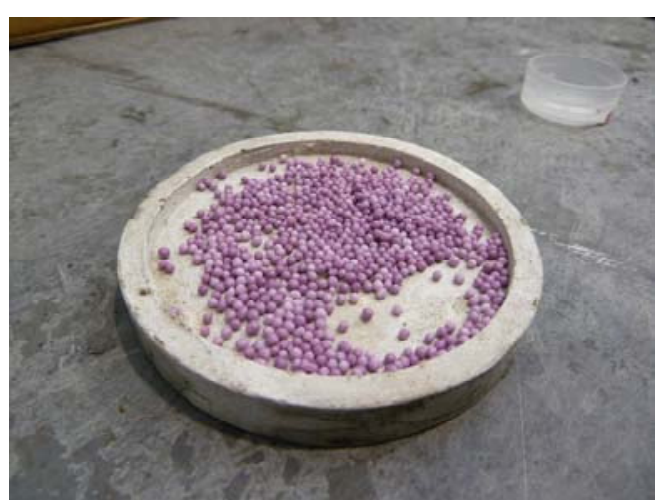

(a)

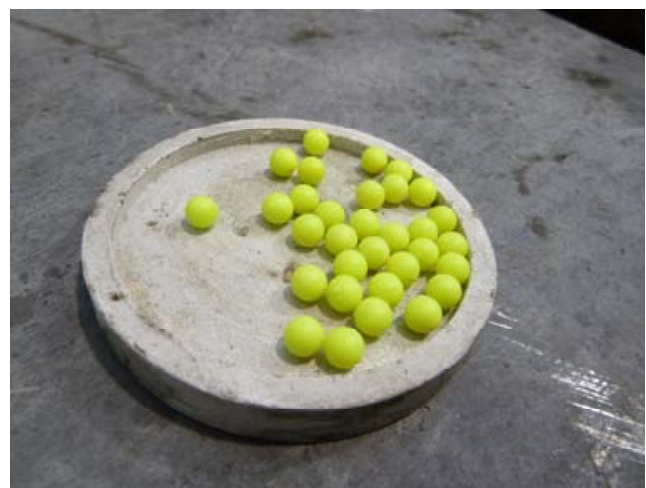

(b)

Figure 1: particles types : (a) sugar particles, (b) plastic spheres.
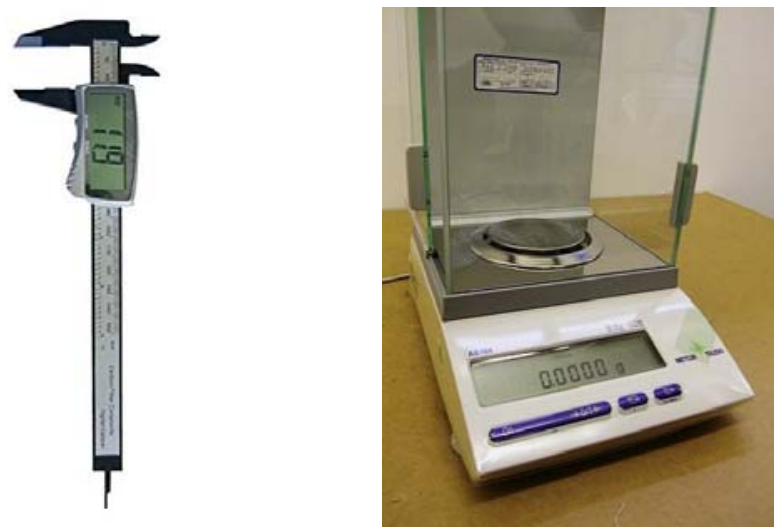

Figure 2: (a) digital calibre and (b) precision balance. 


\subsection{Low Subsonic, Hot Flow Facility}

The set-up used for single particle injection in low-subsonic, medium temperature, carrier flow is shown in Figure 3 (LSHFF facility); the stripper is able to generate an air flow with a volume rate of $0.0025-0.0083\left(\mathrm{~m}^{3} / \mathrm{s}\right)$, in the temperature range $323-923(\mathrm{~K})$. At the exit of the stripper a circular nozzle $8(\mathrm{~mm})$ diameter is mounted, together with an axisymmetric injector perpendicular to the carrier flow velocity (which means that each particle enters the mainstream subjected only to gravity force). The gas is pre-heated before the injection of particles in order to allow the thermocamera to detect their presence. This is not necessary when high-speed jets are considered (high-subsonic cold flow, see the next section) as in that case the temperature difference between particles and the surrounding gas is naturally produced by the conversion of thermal energy into kinetic energy when the gas is accelerated starting form stagnation conditions.

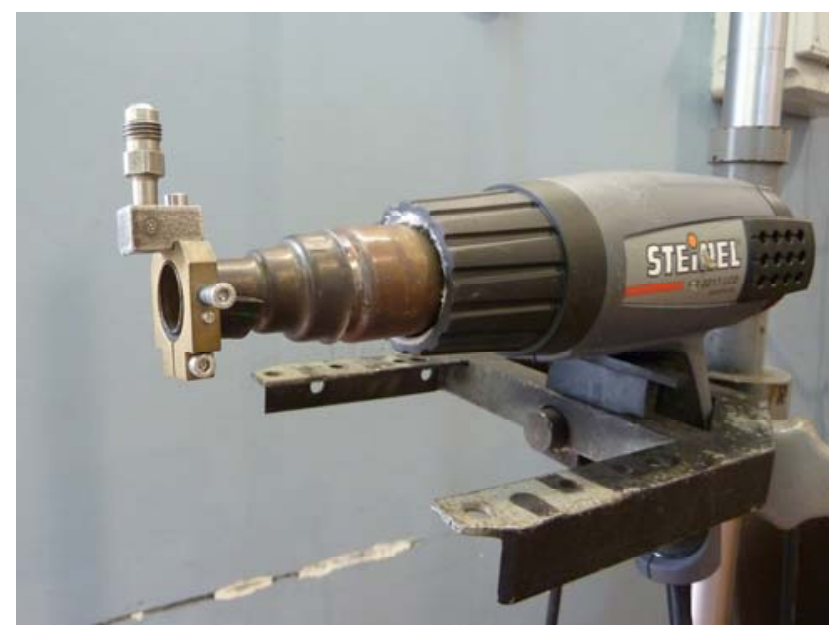

Figure 3: Low Subsonic, Hot Flow Facility.

\section{$\underline{3.3 \text { High Subsonic, Cold Flow Facility }}$}

With this facility (HSCFF) it is possible to generate an axisymmetric free jet driven by a pressure vessel loaded up to $20 \times 10^{5}(\mathrm{~Pa})$. The particles injector is the same employed for the low-subsonic facility, as it can be seen in Fig. 4. By regulating the vessel pressure, it is possible to vary the Mach number of the carrier flow in the range between 0.2 and 0.9, i.e. 68 - $307(\mathrm{~m} / \mathrm{s})$. The flow total temperature is bound at ambient value (the difference between the static temperature of the particle and the total temperature of the carrier flow representing the physical condition allowing particles to be detected by means of a thermocamera). 

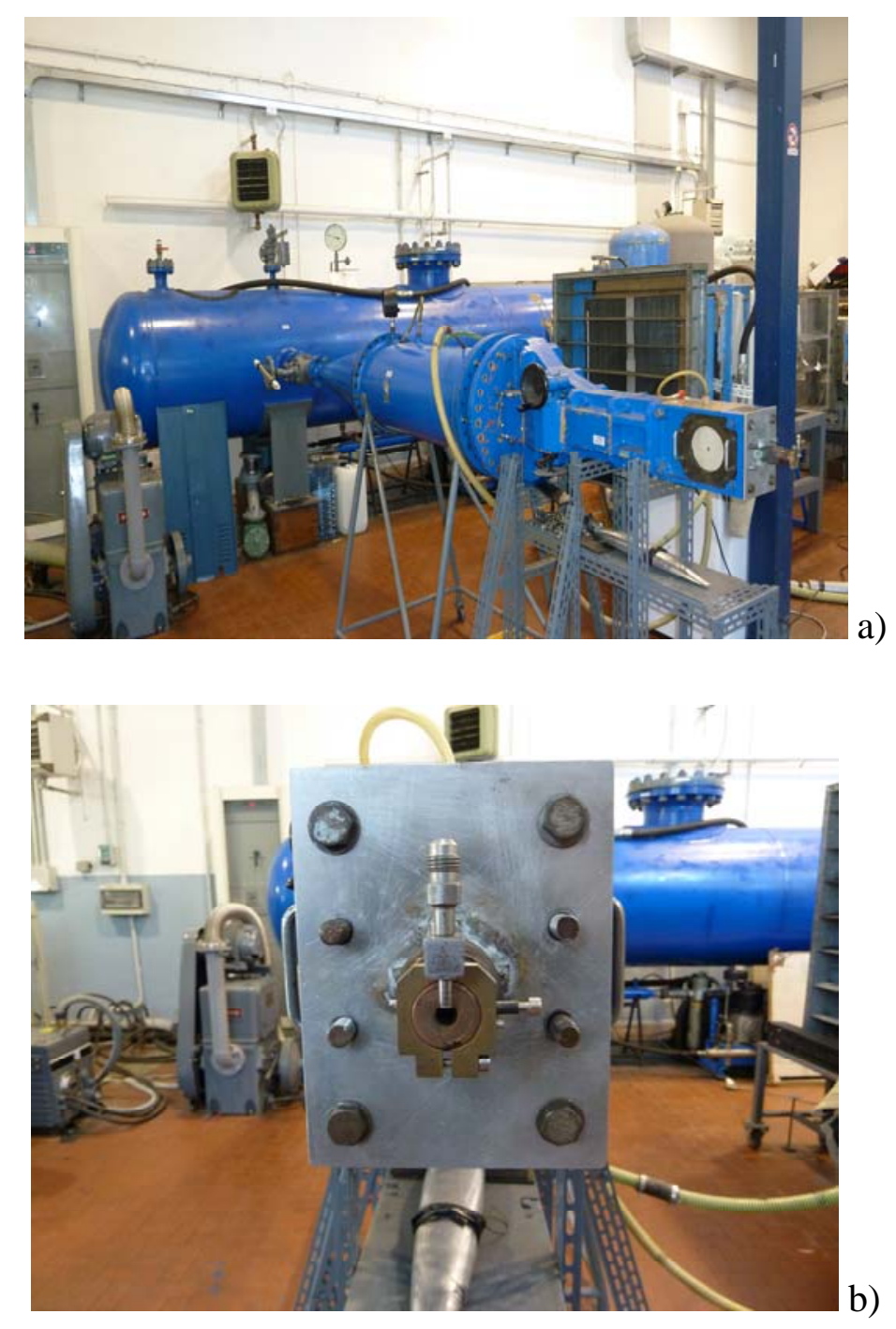

Figure 4: High Subsonic, Cold Flow Facility (a) global view (b) detail of injection zone.

\subsection{Diagnostic Tools and Related Techniques}

(a) Flow characterization

The following approach has been implemented to characterise the flow.

For the LSHFF, the average velocity and temperature at the nozzle exit have been measured using a standard Pitot tube and a "K" thermocouple embedded in the Pitot itself.

For the HSCFF, the total temperature in the stagnation chamber has been measured by means of a thermocouple. Then we have calculated the static flow temperature from standard isentropic tables. A similar procedure has been used to estimate the velocity. First, we have measured the total and 
ambient pressures by means of pressure transducers; then the flow velocity has been computed as a function of the Mach number and the sound velocity.

(b) Infrared System

As anticipated in the introduction, in the present work we have taken an alternative point of view in the analysis of solid particles trajectories in gas flows, based on the application of high velocity infrared thermography. Indeed, to track particles in various conditions, a fast infrared camera FLIR SC6811 (see Fig. 5), with a sensor 640x516 pixels in the midwave band (1.5-5 $\mu \mathrm{m}$ ), has been used. The acquisition frame rate of this camera can be set to $580 \mathrm{~Hz}$ in full frame mode or $16(\mathrm{kHz})$ in the 640x4 pixel mode. The spectral response ranges between 1.5 and 5 (microns). The camera is capable to detect temperature in the range 233 - $2273(\mathrm{~K})$ of black body equivalent with a sensitivity of 20 (mK). A $25 \mathrm{~mm}$ (F2.3) lens has been used to follow the particle trajectory. Integration time and sensor windowing were optimized for particle tracking for a frame rate of 1 000 images/s. For particle tracking, background subtraction was performed in data post-processing to increase the contrast ratio particle/background.

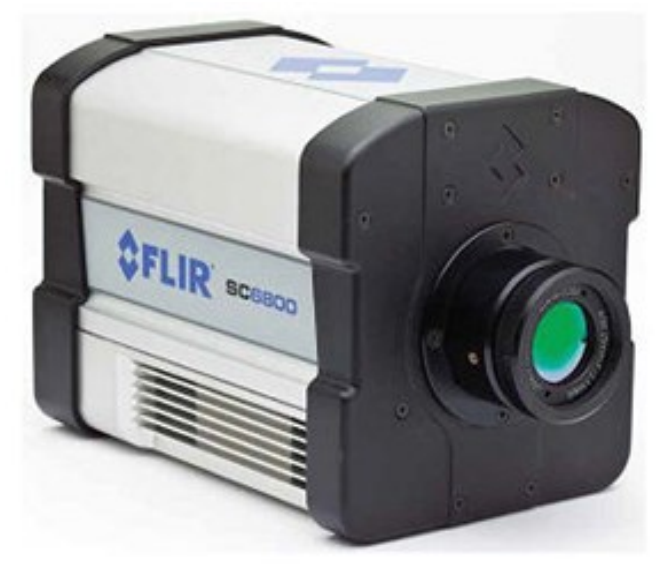

Figure 5: IR camera SC6811.

(c) Particle Trajectories

Particle trajectories have been reconstructed "a posteriori” by using a specific post-processing graphical tool ("Plot Digitizer", a free Java program by which the user is allowed to digitize data points off of scanned plots or orthographic photographs; it also includes an automatic digitization feature that can automatically digitize many types of functional data).

Overall, the repeatability of the experiments was about 95\%. The accuracy for the temperature measurement of the moving particles was $\pm 2 \%$. We found the uncertainty related to the particle position to depend on the gas jet velocity and to be $\cong 5 \%$ for the highest value of the Mach number considered. 


\section{Results}

From a purely physical standpoint, it can be argued that the presence of a jet will obviously act on the inert dispersed phase by accelerating it through momentum exchange. More specifically, as soon as a particle enters the jet (as explained before, it is injected along a direction perpendicular to the jet, the jet being aligned with the horizontal $\mathrm{x}$ direction), its velocity will increases instantaneously across the boundary layer region (very thin for high values of the jet Reynolds number), which separates the jet from the external quiescent environment. Such effect can be essentially ascribed to the drag exerted by the moving gas on the particle, which will transfer momentum from the gas to the solid particle.

The dynamics following the injection of the particle into the jet, however, will also be dependent on other factors, such as the specific "inertia” possessed by a particle, which, as illustrated in Sect. 2, will cause a departure of its trajectory from the path that would be taken by a mass-less and sizeless (ideal) tracer. Also gravity will play an effect in such dynamics. All these effects can be characterized using the quantities defined in Sects. 2.1 and 2.2. Two additional relevant nondimensional parameters can be defined as $l_{x}=\mathrm{L}_{\mathrm{x}} / \mathrm{D}$ and $l_{y}=\mathrm{L}_{\mathrm{y}} / \mathrm{D}$ where $\mathrm{L}_{\mathrm{x}}$ and $\mathrm{L}_{\mathrm{y}}$ are the horizontal distance travelled by the particle before hitting the bottom of the physical domain and the vertical distance of the jet axis from the bottom wall for $\mathrm{x}=0$ (fixed to $9 \mathrm{~cm}$ in the present work).

\subsection{Low Subsonic Hot Flow, Single particle case}

The test conditions we have considered for this case are summarised in Table I.

\begin{tabular}{|l|l|}
\hline Parameter & Value \\
\hline Circular nozzle diameter $[\mathrm{m}]$ & $8 \times 10^{-3}$ \\
\hline Carrier flow velocity [m/s] & 34 \\
\hline Carrier flow Temperature [K] & 323 \\
\hline Sugar Particle Temperature [K] & 288 \\
\hline Carrier Flow Reynolds number $\left(\mathrm{Re}_{\text {JET }}\right)$ & $\cong 1.86 \times 10^{4}$ \\
\hline Mach number $(\mathrm{M})$ & 0.094 \\
\hline Particle Stokes number $(\mathrm{St})$ & $\cong 2.47 \times 10^{-3}$ \\
\hline Froude number $($ Fr) & $\cong 6.5 \times 10^{-3}$ \\
\hline Fluid/particle density ratio $(\xi)$ & 1132 \\
\hline $\mathfrak{R}$ & $\cong 3.56$ \\
\hline
\end{tabular}

Table I: Parameters used for the Low Subsonic Hot Flow, Single particle tests. 
During the experiments, the acquisition frame rate of the thermocamera was set to about $1000(\mathrm{~Hz})$. A $25 \mathrm{~mm} / \mathrm{F} 2.5$ objective was used to follow the trajectory of the spheres.

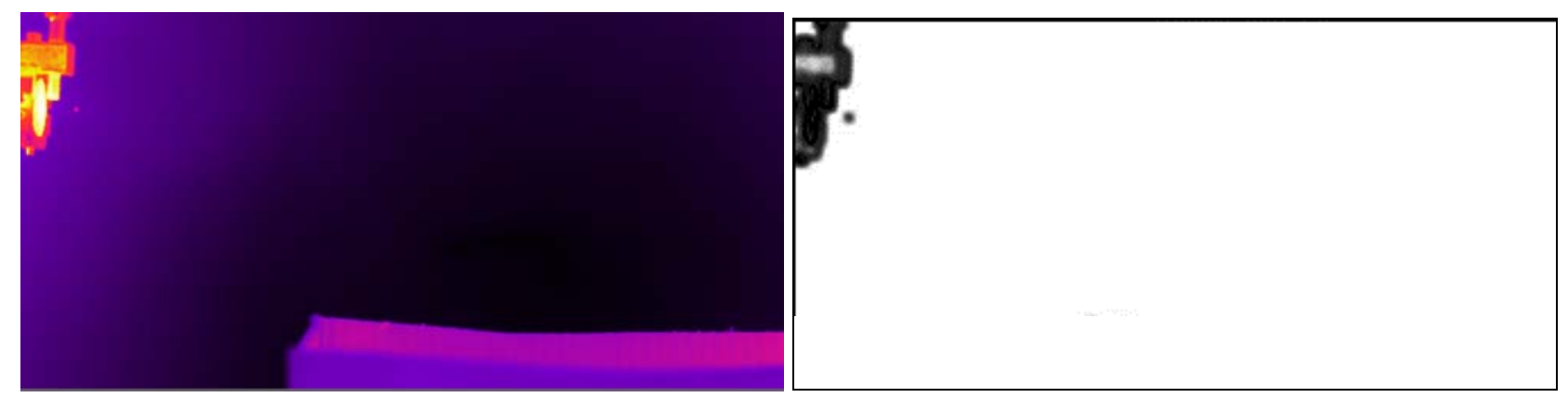

(a) $\mathrm{t}=40.05(\mathrm{~ms})$
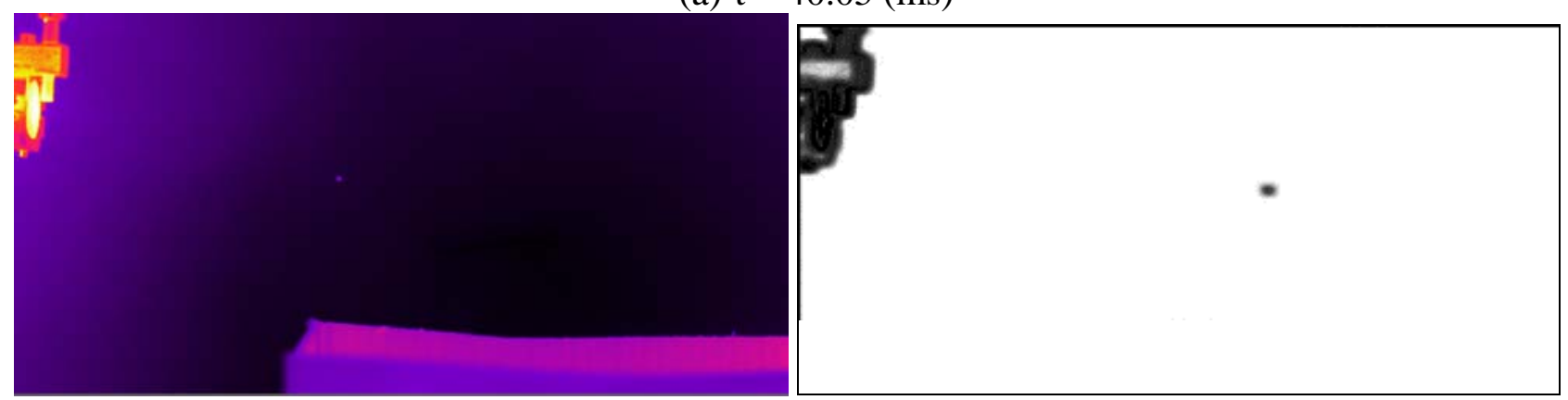

(b) $\mathrm{t}=218.05$ (ms)

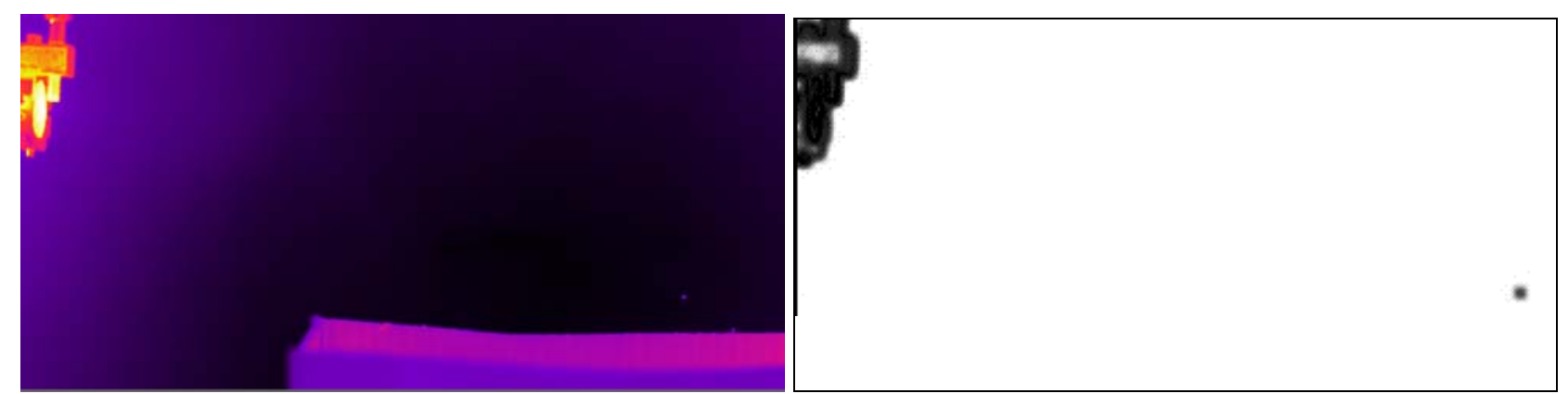

(c) $\mathrm{t}=440.55$ (ms)

Figure 6: Thermographic sequence showing the displacement in time of a single particle of sugar for $\xi=1132, V_{J E T}=34 \mathrm{~m} / \mathrm{s}, T_{J E T}=323 \mathrm{~K}, T_{p}=288 \mathrm{~K}$ (sugar particle initial temperature), $\mathrm{Re}_{\mathrm{JET}} \cong$ $1.86 \times 10^{4}, \mathrm{M}=0.094, \mathrm{R}_{\mathrm{p}}=0.845 \mathrm{~mm}, \mathrm{St} \cong 2.47 \times 10^{-3}, \mathrm{Fr} \cong 6.5 \times 10^{-3}$ (three snapshots at different times, original thermographic picture on the left, digitally enhanced version on the right).

This might be regarded as the most instructive of the cases considered here. Indeed, as shown by the experimental (thermographic) images (Fig. 6) and confirmed by the numerical simulations (Fig. 7), for these conditions, the particle trajectory can ideally be split into three well defined regions according to its slope and curvature (see Fig. 8). Each of these regions, in turn, can be linked to a specific regime of particle motion where different physical effects (particle inertia, weight and drag) have a different relative importance. 


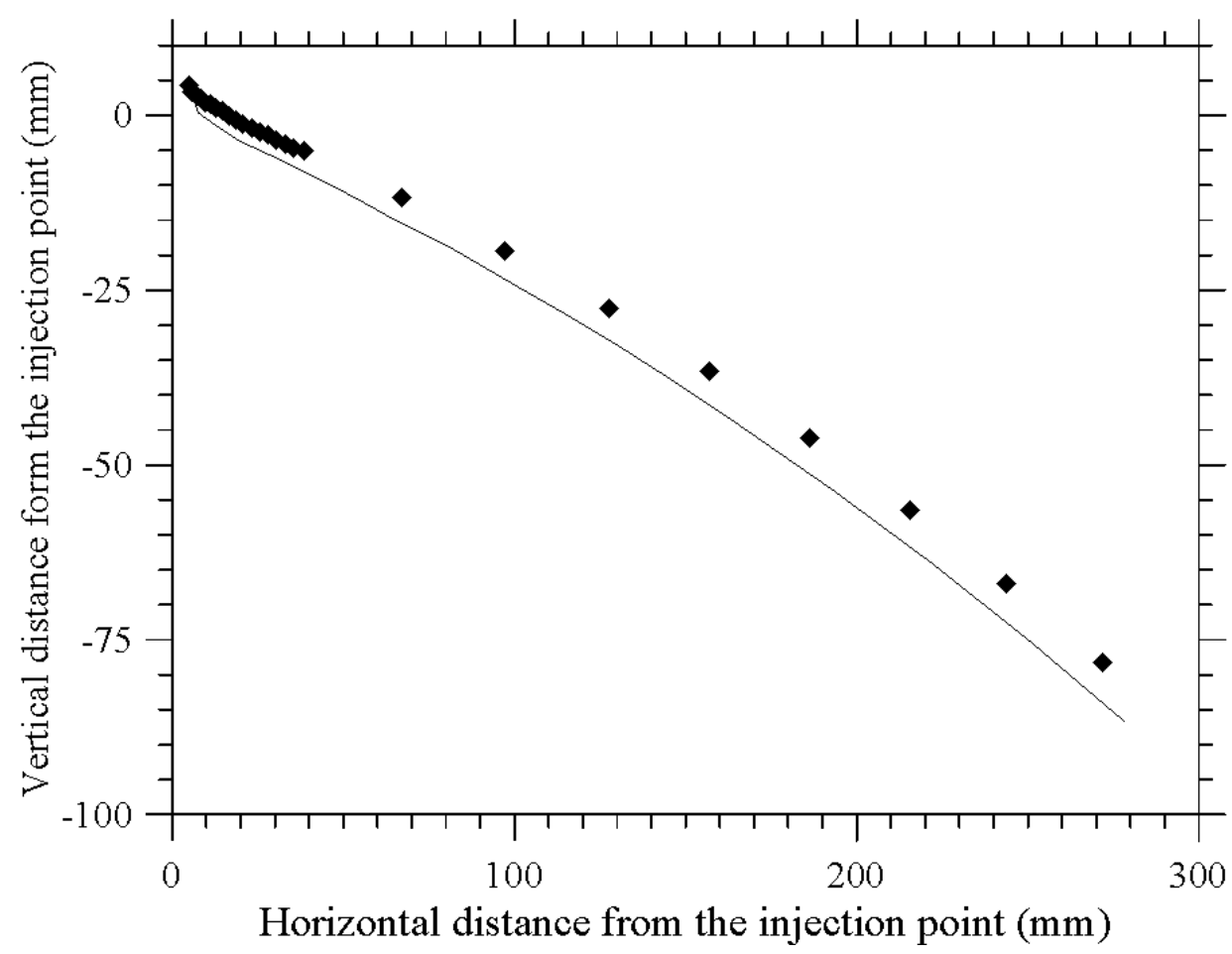

Figure 7: Experimental results (symbols) vs Numerical simulation (solid line) for the same conditions considered in Fig. 6.

For $0<\mathrm{x}<2 \mathrm{~mm}$, i.e. in proximity to the particle injector, in particular, the slope of the trajectory is relatively steep (locally concave upward), which clearly indicates the dominant role played there by gravity in determining the particle dynamics (obviously, without the disturbing effect exerted on it by the horizontal "forced" flow, the particle would ideally move along a purely vertical trajectory with its velocity theoretically approaching the asymptotic state represented by eq. (8)). However, since after a few milliseconds the particle crosses the boundary layer separating the jet from the external quiescent environment, the trajectory suddenly takes a different slope and curvature.

As evident in Fig. 7, as soon as the particle moves beyond the position $x=20 \mathrm{~mm}$, the slope of its trajectory undergoes a significant decrease while the concavity changes from upward to downward. Both these effects can be regarded as the manifestation of a significant modification occurring in the physical mechanisms governing the particle motion. Indeed, while in the first stage of evolution particle dynamics can be described essentially in terms of a balance between inertia and gravity, in the second stage (particle inside the jet) another important force starts to play a significant role, i.e. the particle drag due to its interaction with the high-velocity gas (see the central region in Fig. 8).

When the particle (due to the downward component of its velocity) leaves the jet, the second stage finally decades into a less energetic regime due to the discontinuation of the jet-induced horizontal particle propelling action. This final regime (sketched in Fig. 8 as “regime 3”) can be identified via the corresponding variation in the slope of the trajectory which slightly increases beyond $\mathrm{x} \cong 100 \mathrm{~mm}$ (while its concavity does not change). 


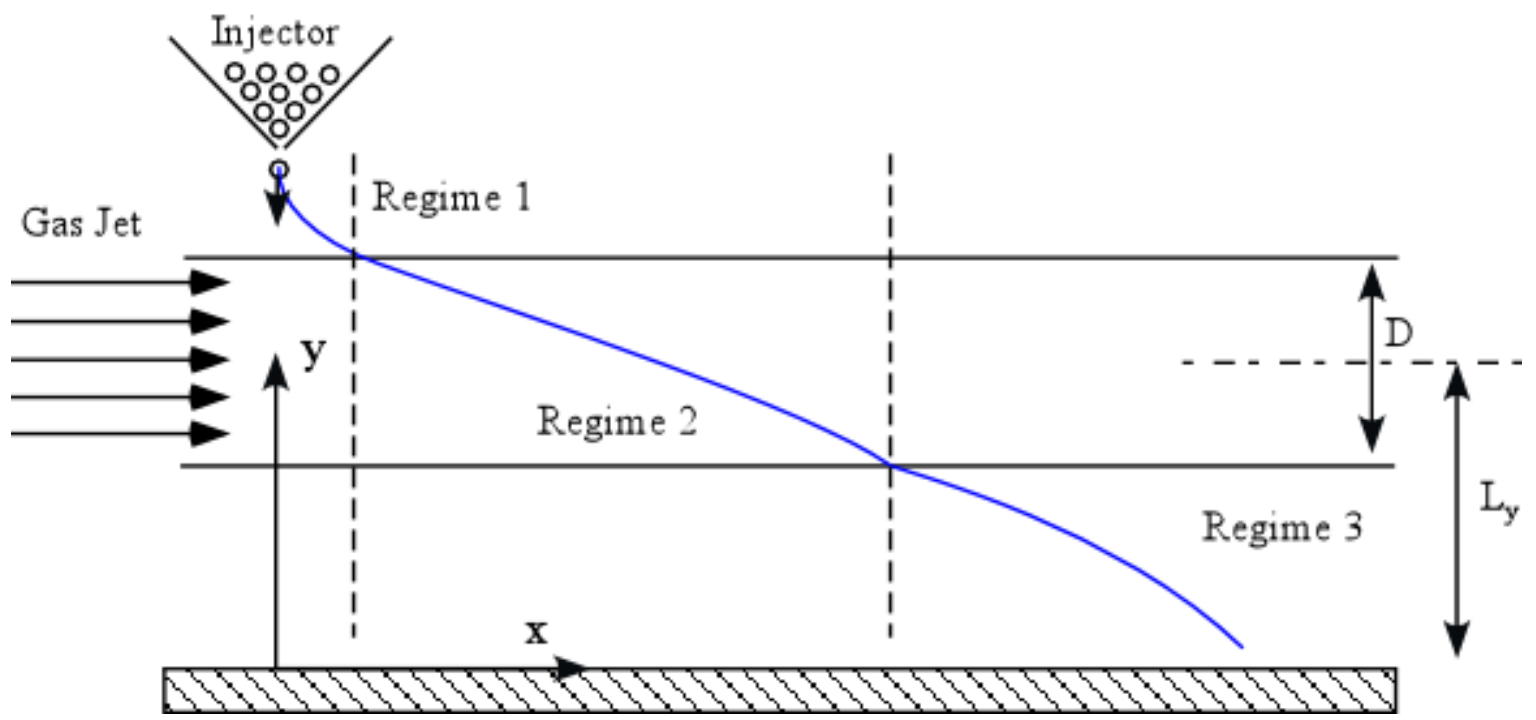

Figure 8: Sketch of the different stages of evolution undergone by the particle during its motion from the injector towards the bottom wall.

\subsection{Low Subsonic Hot Flow, Particle swarm case}

Given the good agreement between the experiments and the numerical simulations for $\mathrm{M}=0.094$, towards the end to test further the flexibility of the computational framework and its ability to deal with a variety of circumstances, we have considered other situations with increasing complexity. In particular, keeping fixed the particle material ( $\xi=1132$ for sugar particles), we have increased the Mach number in order to make the fluid compressibility significant and varied parametrically the Stokes number to assess the influence of particle size. The outcomes of these simulations, summarised in Fig. 9, indicate that while for a fixed $S t$ an increase in the Mach number can delay the particle settling time and allow it to be transported over a larger distance, making the Stokes number higher (at fixed $\mathrm{M}$ ) produces exactly an opposite trend (it causes a significant decrease in the distance covered by the particle before it hits the bottom; this trend reflecting the interpretation we have given before about the meaning of the parameter $\mathfrak{R}$ ).

Experiments for the same particles considered in Sect. 4.1 (Radius $\mathrm{R}_{\mathrm{p}}=0.845 \mathrm{~mm}, S t \cong 2.47 \times 10^{-3}$ ) and $M=0.65$ (corresponding in the space of parameters to the top branch visible in Fig. 9) are shown in Fig. 10 (the reader being referred to Table II for the related experimental conditions). 


\begin{tabular}{|l|l|}
\hline Parameter & Value \\
\hline Circular nozzle diameter $[\mathrm{m}]$ & $8 \times 10^{-3}$ \\
\hline Carrier flow velocity [m/s] & 234 \\
\hline Carrier flow Temperature [K] & 323 \\
\hline Sugar Particle Temperature [K] & 288 \\
\hline Carrier Flow Reynolds number $\left(\mathrm{Re}_{\mathrm{JET}}\right)$ & $\cong 1.28 \times 10^{5}$ \\
\hline Mach number $(\mathrm{M})$ & 0.65 \\
\hline Particle Stokes number $(\mathrm{St})$ & $\cong 2.47 \times 10^{-3}$ \\
\hline Froude number $($ Fr) & $\cong 6.5 \times 10^{-3}$ \\
\hline Fluid/particle density ratio $(\xi)$ & 1132 \\
\hline $\mathfrak{R}$ & $\cong 0.52$ \\
\hline
\end{tabular}

Table II: Parameters used for the Low Subsonic Hot Flow, Particle swarm tests.

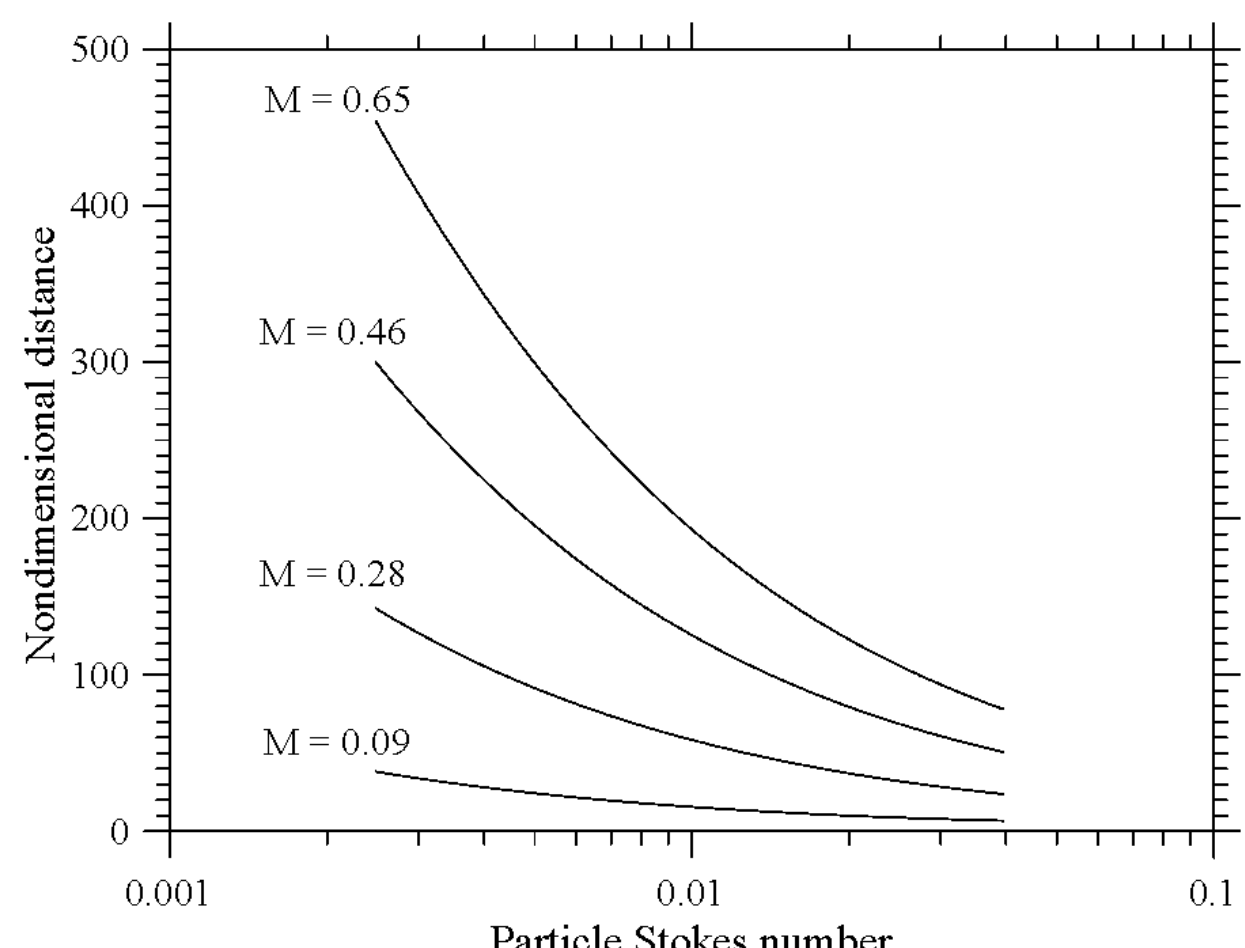

Figure 9: Nondimensional horizontal distance $\left(l_{x}=\mathrm{L}_{\mathrm{X}} / \mathrm{D}, \mathrm{D}=8 \mathrm{~mm}\right)$ covered by the particle $(\xi=$ $1132, F r \cong 6.5 \times 10^{-3}$ ) before hitting the bottom wall (located at a nondimensional distance from the jet axis $l_{y}=\mathrm{L}_{\mathrm{y}} / \mathrm{D}=11$ ) as a function of the Stokes number for different values of the jet Mach number (numerical results, the considered range of values of the Stokes number corresponds to the particle radius spanning the dimensional interval $0.845 \leq \mathrm{R}_{\mathrm{p}} \leq 3.38 \mathrm{~mm}$ ). 
Moreover, for this experiment, in place of a single particle we have expressly considered a swarm in order to get some statistically meaningful information about the influence exerted on the swarm dynamics by the non-constant particle size as mentioned at the end of Sect. 3.1. Such particles have yet been injected into the main jet in a direction perpendicular to it (using gravity as the force naturally forcing particles to move backward). Equivalent numerical simulations have been carried out introducing a certain degree of uncertainty in the problem by allowing the particle size to vary in a certain range (cantered on the nominal value indicated in Sect. 3.1, i.e. diameter $1.69 \times 10^{-3}(\mathrm{~m})$ with amplitude 20\%).

In this way the response of the algorithm has been assessed with respect to more realistic situations in which many particles may be present in the jet, each displaying a potentially different behaviour according to its inertial (size and mass) properties.

Moreover, with the explicit intent to explore the role of inertial effects with respect to both the horizontal and vertical directions, for these additional tests we have considered (both numerically and experimentally) a jet slightly inclined with respect to the horizontal direction (positive inclination angle $\theta \cong 1^{\circ}$ ).
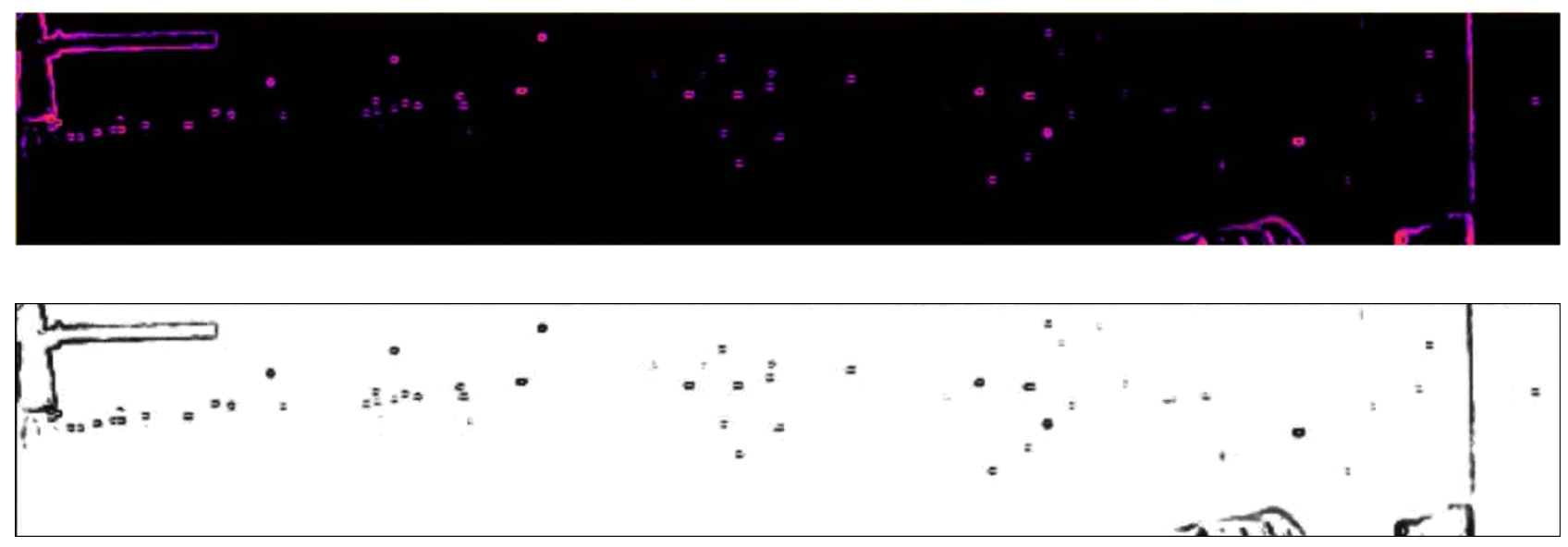

Figure 10: Snapshot showing the distribution in space of particles for $\xi=1132, V_{J E T}=234 \mathrm{~m} / \mathrm{s}$, $T_{J E T}=288 \mathrm{~K}, T_{p}=288 \mathrm{~K}$ (sugar particle initial temperature), $\mathrm{Re}_{\mathrm{JET}} \cong 1.28 \times 10^{5}, \mathrm{M}=0.65, \mathrm{R}_{\mathrm{p}}=0.845$ $\mathrm{mm}, S t \cong 2.47 \times 10^{-3}, F r \cong 6.5 \times 10^{-3}:$ a) original thermographic picture b) digitally enhanced version.

As shown by both experimental (Fig. 10) and numerical (Fig. 11) results, the paths taken by the particles can be split into two main categories.

Indeed, while some particles display a behaviour qualitatively similar to that presented in Fig. 7 (undergoing displacement towards the bottom as time increases), other particles are subjected to a lift effect, which tends to propel them upwards. 


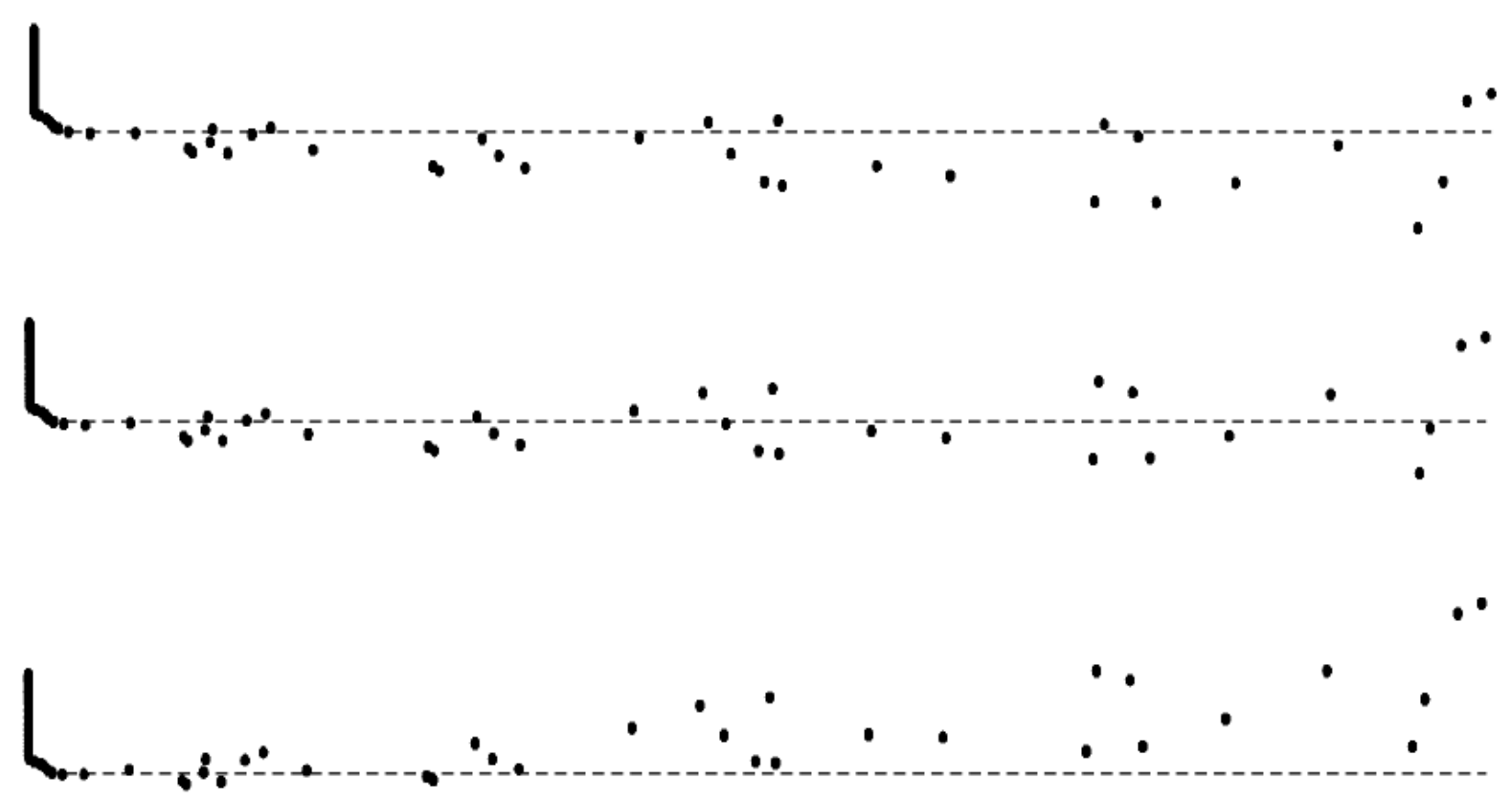

a)

b)

Figure 11: Numerical simulation of particle swarm dynamics for the same conditions considered in Fig. 9 and different inclinations of the jet: a) $\theta=0.7^{\circ}$, b) $\theta=1^{\circ}$, c) $\theta=1.5^{\circ}$.

This second effect, which has to be ascribed to the (positive) vertical component of the jet velocity, (which, by exerting an upwards-directed drag on the particles, counteracts the effect of gravity), clearly depends on the considered inclination of the jet and the particle size (leading to a different inertial response). As the reader will realise by taking a look at Fig. 11, while for $\theta=0.7^{\circ}$ most of particles (as they travel from the left side to the right side of the field of view) fall under the ideal horizontal line passing through the injection point, for $\theta=1.5^{\circ}$ this behaviour is reversed with most of particles lying above this line. Notably for $\theta=1.0^{\circ}$ the amount of particles located above and below this line is more or less the same in good agreement with the experiments (Fig. 10).

The above qualitative arguments are quantitatively supported by the data shown in Fig. 12.

As $F r$ does not depend on the particle size, the different behaviours displayed by particles in this case must indeed be explained according to the different values of $S t$. The larger the particle radius, the higher the value taken by this number, which indicates that smaller particles will tend to "feel" the upward propulsive action exerted on them by the jet much better than particles with larger diameter (this effect can clearly be appreciated in Fig. 12 where the non-dimensional parameter $l_{\mathrm{x}}$ has been reported as a function of the particle Stokes number for different inclinations of the jet). It can be seen that for $\theta=1.0^{\circ}$ a variation of $20 \%$ of the particle size with respect to its nominal value can produce a change in the parameter $l_{x} \cong 25 \%$.

By providing information about the influence of both the jet inclination angle and the size of the particle, this plot can be used as a relevant basis for the interpretation (in a statistical sense) of the "scattered" vertical distribution of particles in Figs. 10 and 11. 


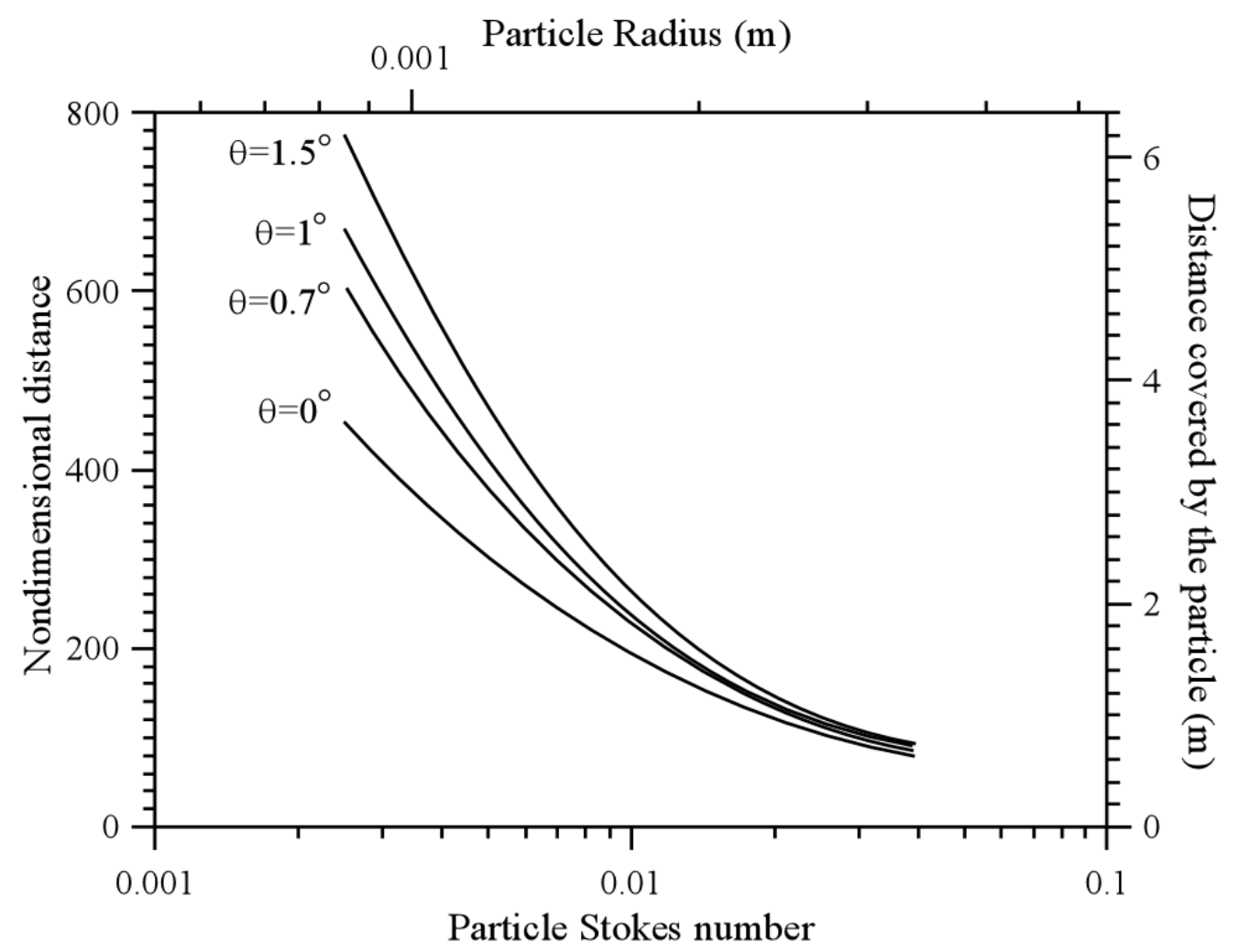

Figure 12: Nondimensional horizontal distance $\left(l_{x}=\mathrm{L}_{x} / \mathrm{D}, \mathrm{D}=8 \mathrm{~mm}\right)$ covered by the particle before hitting the bottom wall (located at a nondimensional distance from the jet axis $l_{y}=\mathrm{L}_{\mathrm{y}} / \mathrm{D}=11, \mathrm{~L}_{\mathrm{y}}=90$ $\mathrm{mm}$ ) as a function of the Stokes number for $\xi=1132, F r \cong 6.5 \times 10^{-3}, \mathrm{M}=0.65$ and different values of the jet inclination angle (numerical results).

\subsection{High Subsonic Cold Flow, Single particle}

As in the experiments described in the preceding section we have varied the jet Mach number while keeping constant the particle Stokes and Froude numbers and the density ratio, in the remainder of this study we consider a final (experimental) example for which we change the particle radius ( $S t$ ), its material $(\xi)$ and the diameter of the jet $\left(l_{y}\right)$ while keeping fixed the distance of the injection point from the bottom.

Moreover, we further increase the compressibility of the flow by considering a value of the Mach number close to 0.9 (see Table III for the considered experimental conditions). 


\begin{tabular}{|l|l|}
\hline Parameter & Value \\
\hline Circular nozzle diameter [m] & $2 \times 10^{-2}$ \\
\hline Carrier flow velocity [m/s] & 308 \\
\hline Carrier flow Temperature [K] & 288 \\
\hline Plastic Particle Temperature [K] & 288 \\
\hline Carrier Flow Reynolds number $\left(\mathrm{Re}_{\mathrm{JET}}\right)$ & $\cong 4.22 \times 10^{5}$ \\
\hline Mach number $(\mathrm{M})$ & 0.9 \\
\hline Particle Stokes number $(\mathrm{St})$ & $\cong 5 \times 10^{-3}$ \\
\hline Froude number $($ Fr) & $\cong 1.65 \times 10^{-3}$ \\
\hline Fluid/particle density ratio $(\xi)$ & 1078 \\
\hline $\mathfrak{R}$ & $\cong 4.69$ \\
\hline
\end{tabular}

Table III: Parameters used for the High Subsonic Cold Flow, Single particle tests.

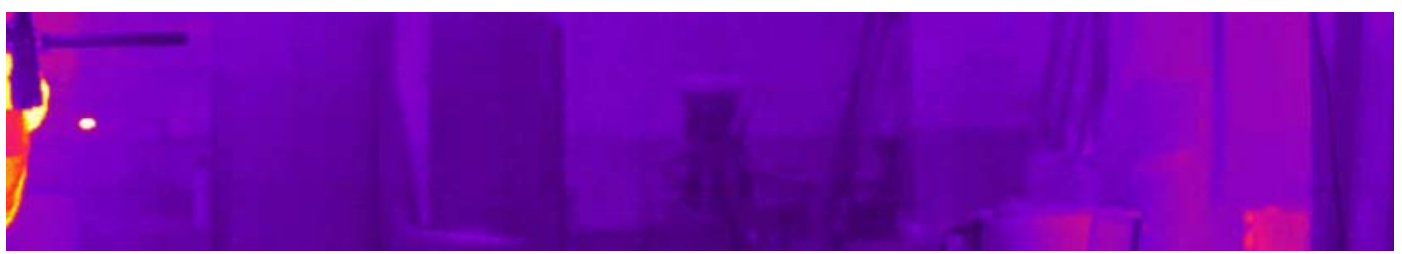

(a) $\mathrm{t}=15(\mathrm{~ms})$

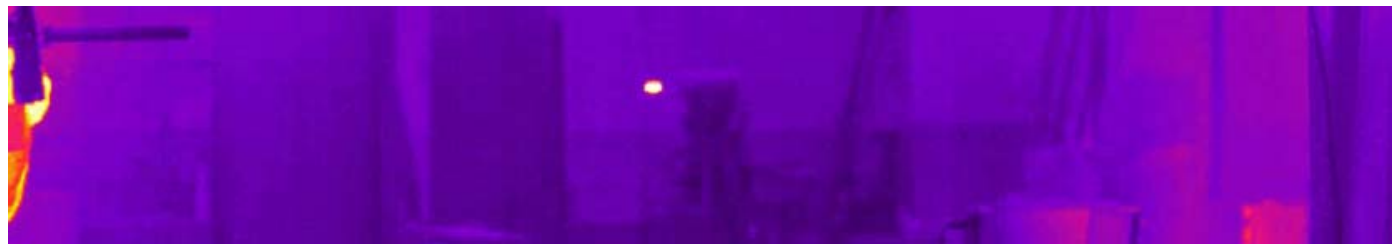

(b) $\mathrm{t}=25$ (ms)

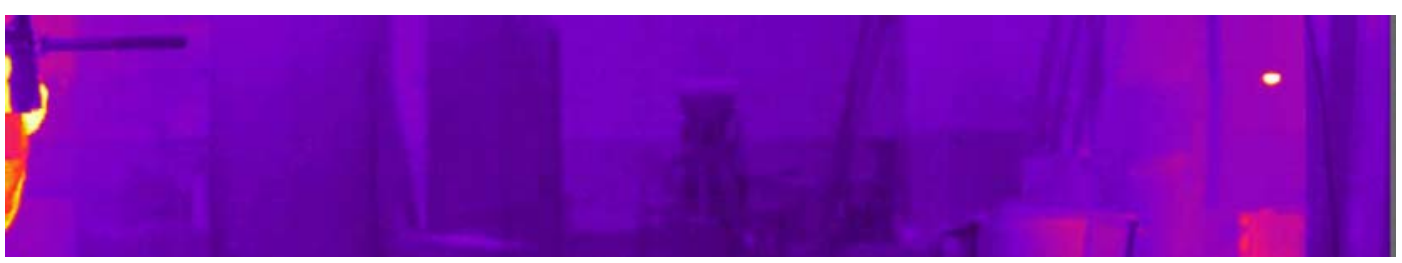

(c) $\mathrm{t}=50$ (ms)

Figure 13: Thermographic sequence showing the displacement in time of a single particle of plastic for $\xi=1078, V_{J E T}=308 \mathrm{~m} / \mathrm{s}, T_{J E T}=288 \mathrm{~K}, T_{p}=288 \mathrm{~K}$ (plastic particle initial temperature), $\operatorname{Re}_{J E T} \cong$ $4.22 \times 10^{5}, \mathrm{M}=0.9, \mathrm{R}_{\mathrm{p}}=3 \mathrm{~mm}, \mathrm{St} \cong 5 \times 10^{-3}, \mathrm{Fr} \cong 1.65 \times 10^{-3}$ (three snapshots at different times, original thermographic picture on the left, digitally enhanced version on the right). 

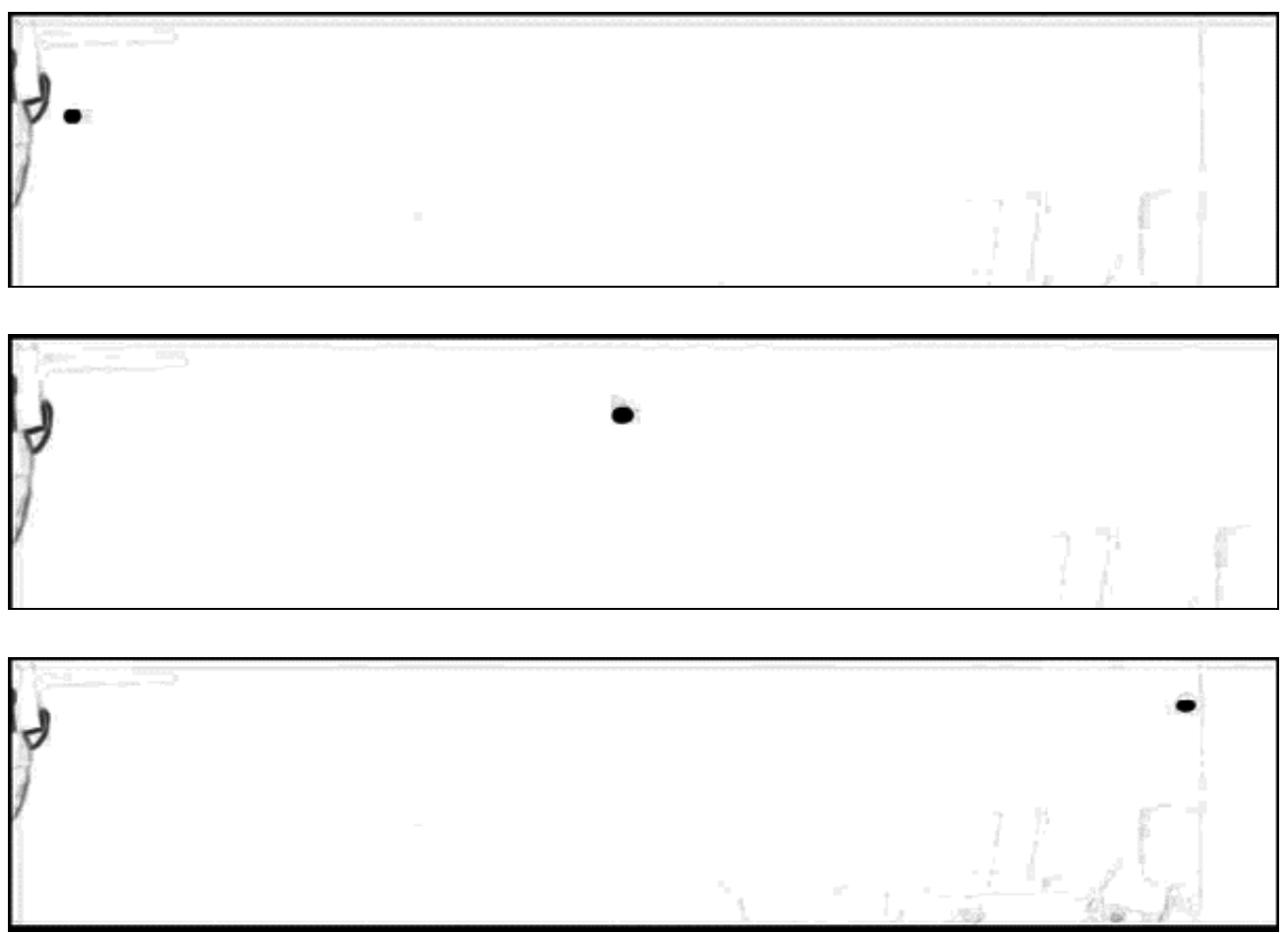

Figure 14: Thermographic Sequence (digitally enhanced pictures) for the same conditions considered in Fig. 13.

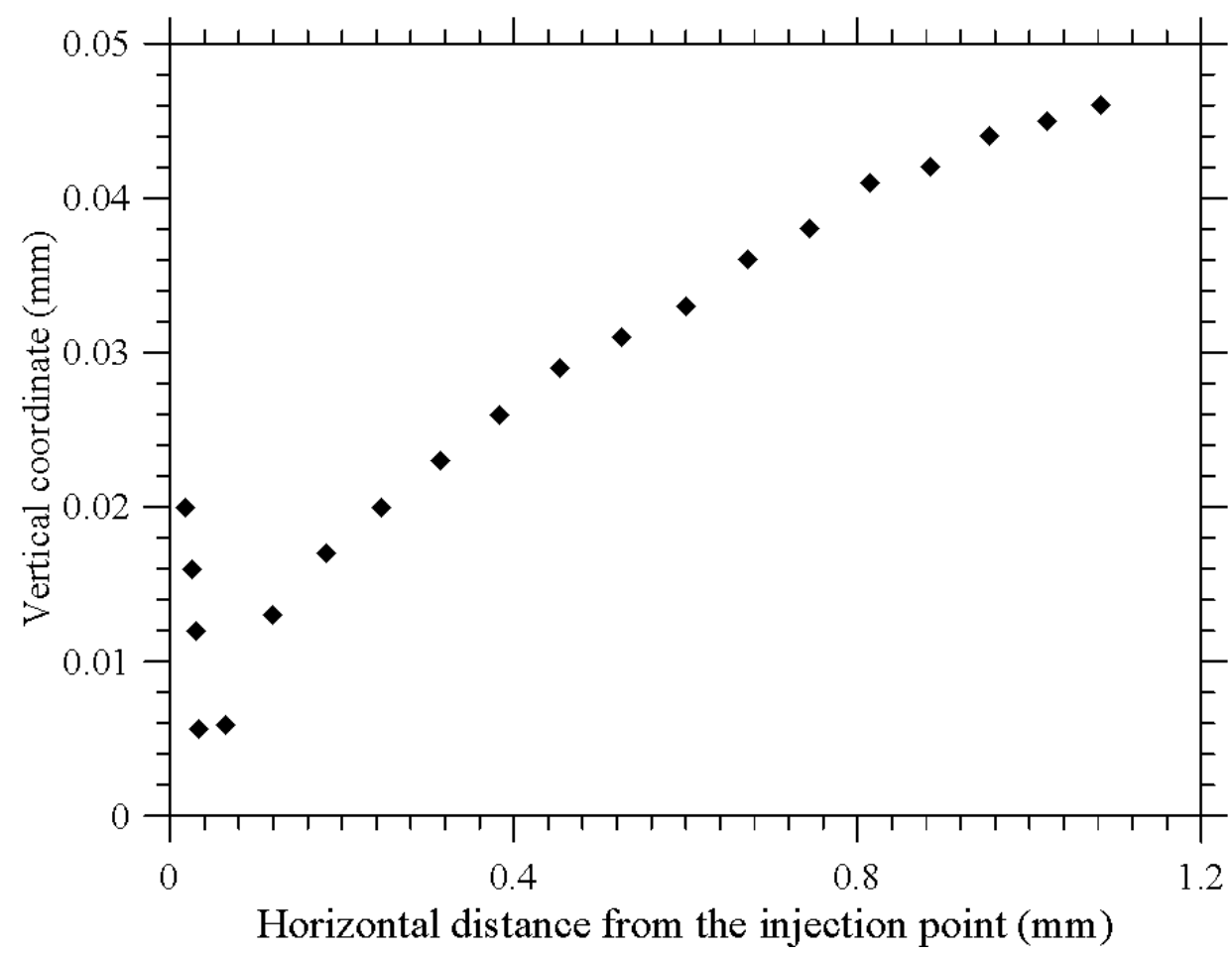

Figure 15: Experimental particle trajectory for the same conditions considered in Fig. 13. 


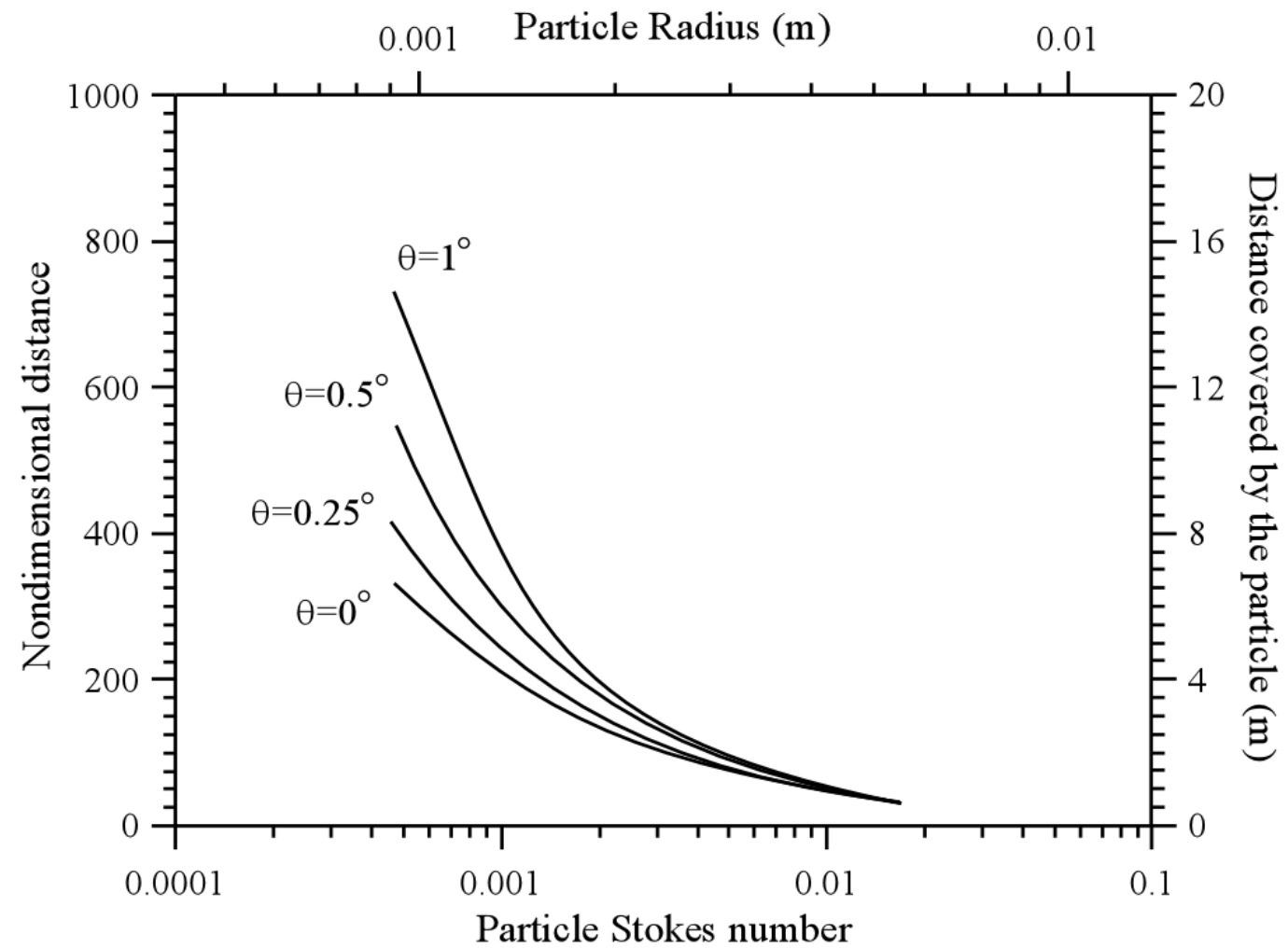

Figure 16: Nondimensional horizontal distance $\left(l_{x}=\mathrm{L}_{X} / \mathrm{D}, \mathrm{D}=20 \mathrm{~mm}\right)$ covered by the particle before hitting the bottom wall (located at a nondimensional distance from the jet axis $l_{y}=\mathrm{L}_{\mathrm{y}} / \mathrm{D}=4.5$, $\mathrm{L}_{\mathrm{y}}=90 \mathrm{~mm}$ ) as a function of the Stokes number for $\xi=1078, \mathrm{Fr} \cong 1.65 \times 10^{-3}, \mathrm{M}=0.9$ and different values of the jet inclination angle.

The experiments shown in Figs. 13-15 (yet for the case with the jet inclined by $1^{\circ}$ ) confirm that even if the particle radius is significantly increased, the jet lifting action (due to its inclination) exerted on the particle can still be significant, provided the Mach number becomes higher and the jet cross-sectional area is increased (corresponding to a decrease in $l_{y}$ ).

This conclusion is further reinforced by the expanded set of (numerically computed) data summarised in Fig. 16.

While the nondimensional distance $l_{x}$ tends ideally to infinite in the limit as the particle Stokes number tends to zero (limiting situation in which a particle would behave as a mass-less and sizeless tracer experiencing neither gravity nor other inertial forces), it is shown that for a real (inertial) particle, an increase in the inclination angle can greatly delay the particle settling process, this effect being amplified for increasing values of $\mathrm{M}$. Another conclusion stemming from such observations is that, for fixed particle Stokes number and jet inclination (compare Fig. 12 and Fig. 16), the higher the considered jet velocity (Mach number) the more sensitive the particle dynamics become with respect to the jet inclination. 


\section{Conclusions}

The good agreement between experimental and numerical results indicates that the use of thermography is a "viable" option for the tracking of high-velocity particles in compressible flow. We wish to re-emphasize that no particular care has been taken to make the particles "visible" (i.e. by making them fluorescent or temperature sensitive), our particles being simple inert quasispherical sugar spheres or plastic spheres. By using a fast infrared camera we could successfully track the motion of all these particles (producing accurate data sets to represent their trajectories), regardless of their size, mass and surface properties (i.e. material) in different flow regimes.

This approach was successfully coupled with numerical simulations based on a hybrid formulation in which the balance equations for the fluid flow were solved in the framework of a classical van Leer splitting (over a uniform mesh), whereas the particles were tracked separately in the context of a Lagrangian approach. Owing to the negligible mass load (a single particle or a limited set of particles being injected in the gas flow), the coupling between the Eulerian and Lagrangian approaches has been limited to a one-way interaction, i.e. the back influence of the particle on the surrounding fluid flow has been neglected.

Comparison between numerical and experimental results has been instrumental, on the one hand, in demonstrating that thermography can be seen as a valuable strategy for the investigation of these subjects (potentially providing details comparable for quality and precision to those which can be obtained in the framework of numerical simulations), and, on the other hand, in proving that the present combined Eulerian-Lagrangian numerical formulation is robust and sufficiently flexible to be used in relatively wide regions of the space of parameters. Accordingly, numerical simulations have expressly been used to expand the original set of conditions considered for the experiments (limited to few cases in terms of particle sizes and jet properties). By allowing the problem characteristic numbers to span relatively wide ranges, detailed maps have been produced revealing the particle behaviour in the space of parameters, which proved very helpful in interpreting the effects produced by uncertainties (such as jet inclination angle and particle size distribution) often hidden to experimental analysis.

By virtue of such a process of "abstraction" we could build a more general framework in which our experimental findings were just realizations. These attempts may be regarded as a classical example in which numerical and experimental activities can support each other by enabling insights which would be otherwise out of reach.

Future work will be devoted to extend these studies to the case of multiple particles being injected into the gas jet covering regimes which range from the case of dilute systems to situations with a particle concentration is so high that a two-way coupling strategy must necessarily be implemented. 


\section{References}

[1] Scala F., (2013), Particle-fluid mass transfer in multiparticle systems at low Reynolds numbers, Chemical Engineering Science, 91, 90-101.

[2] Lappa M., Drikakis D., Kokkinakis I., (2017), On the propagation and multiple reflections of a blast wave travelling through a dusty gas in a closed box, Phys. Fluids, 29(3), 033301 (19 pages).

[3] Yang N., Wang W., Ge W., Li J., (2003), CFD simulation of concurrent-up gas-solid flow in circulating fluidized beds with structure-dependent drag coefficient, Chemical Engineering Journal , 96(1-3), 71-80.

[4] Scala F., (2018), Particle agglomeration during fluidized bed combustion: Mechanisms, early detection and possible countermeasures, Fuel Processing Technology, 171, 31-38.

[5] Cortés C. and Gil A., (2007), Modelling the gas and particle flow inside cyclone separators Progress in Energy and Combustion Science, 33(5), 409-452.

[6] Rodnianski V., Krakauer N., Darwesh K., Levy A., Kalman H., Peyron I., Ricard F., (2013), Aerodynamic classification in a spiral jet mill, Powder Technology , 243, 110-119.

[7] Molerus O., (1996), Overview: Pneumatic transport of solids, Powder Technology, 88(3) 309321.

[8] Thimothée R., Chauveau C., Halter F., Gökalp I., (2016), Experimental Investigation of the Mechanisms of Cellular Instabilities Developing on Spherical Two-Phase Flames, Combustion Science and Technology, 188(11), 2026-2043.

[9] Kuo K.K., (1996), Recent Advances in Spray Combustion: Spray Combustion Measurements and Model Simulation, Progress in Astronautics and Aeronautics, ISBN: 978-1-56347-181-0, 1996 Published by American Institute of Aeronautics and Astronautics https://doi.org/10.2514/4.866432 [10] Aleiferis P.G. and van Romunde Z.R., (2013), An analysis of spray development with isooctane, n-pentane, gasoline, ethanol and n-butanol from a multi-hole injector under hot fuel conditions, Fuel, 105, 143-168

[11] Heimann R.B., (1996), Plasma-Spray Coating: Principles and Applications, VCH, Berlin, 1996.

[12] Pasandideh-Fard M., Pershin V., Chandra S., Mostaghimi J., (2002), Splat shapes in a thermal spray coating process: Simulations and experiments, Journal of Thermal Spray Technology, 11(2) 206-217.

[13] Ukai, T., Zare-Behtash, H. and Kontis, K. (2017) Suspended liquid particle disturbance on laser-induced blast wave and low density distribution, Phys. Fluids, 29(12), 126104.

[14] Chen L. Yong S.Z, Ghoniem A.F., (2012), Oxy-fuel combustion of pulverized coal: Characterization, fundamentals, stabilization and CFD modelling, Progress in Energy and Combustion Science, 38(2), 156-214.

[15] Lomba R., Bernard S., Gillard P., Mounaım-Rousselle C., Halter F., Chauveau C., Tahtouh T. and Guezet O., (2016), Comparison of Combustion Characteristics of Magnesium and Aluminum Powders, Journal Combustion Science and Technology, 188(11-12), 1857-1877. 
[16] Najjar F. M., Ferry J. P., Haselbacher A. and Balachandar S., (2006), Simulations of SolidPropellant Rockets: Effects of Aluminum Droplet Size Distribution, Journal of Spacecrafts and Rockets, 43(6), 1258-1270.

[17] Lappa M., (2018), On the transport, segregation and dispersion of heavy and light particles interacting with rising thermal plumes, Phys. Fluids, 30(3), 033302 (23 pages).

[18] McGrattan K.B., Baum H.R, and Rehm R.G., (1998), Large eddy simulations of smoke movement, Fire Safety Journal, 30, 161-178.

[19] Kissane M.P., (2008), On the nature of aerosols produced during a severe accident of a watercooled nuclear reactor, Nucl. Eng. Des., 238, 2792-2800.

[20] Valentine G.A. and Wohletz K.H., (1989), Numerical Models of Plinian Eruption Columns and Pyroclastic Flows, Journal of Geophysical Research, 94(B2), 1867-1887

[21] Haszpra T. and Tél T., (2011), Volcanic ash in the free atmosphere: A dynamical systems approach, Journal of Physics: Conference Series 333, 012008 (12 pages).

[22] De Cock N., Massinon M., Salah S.O.T. and Lebeau F., (2017), Investigation on optimal spray properties for ground based agricultural applications using deposition and retention models, Biosystems Engineering, 162, 99 - 111.

[23] Yang C.T., (1977), The movement of sediment in rivers, Geophysical Surveys, 3(1), 39-68.

[24] Perkins R.J., Ghosh S., Phillips J.C. (1991) The Interaction Between Particles and Coherent Structures in a Plane Turbulent Jet. In: Johansson A.V., Alfredsson P.H. (eds) Advances in Turbulence 3. Springer, Berlin, Heidelberg.

[25] Kennedy I.M and Moody M.H., (1998), Particle dispersion in a turbulent round jet, Experimental Thermal and Fluid Science 18, 11-26.

[26] Decker R. K., Meier H. F., Fritsching U., Mori M. , (2010), Analysis of Particle Interaction with Coherent Structures in a Two-phase Mixing Jet, Multiphase Science and Technology, 22(1), 130, DOI: 10.1615/MultScienTechn.v22.i1.10

[27] Tsuji T., Miyauchi T., Oh S., Tanaka T., (2010), Simultaneous Measurement of Particle Motion and Temperature in Two-Dimensional Fluidized Bed with Heat Transfer, KONA Powder and Particle Journal, 28, 167-179.

[28] Charogiannis A., Zadrazil I., Markides C.N., (2016), Thermographic particle velocimetry (TPV) for simultaneous interfacial temperature and velocity measurements, Int. J. Heat Mass Transfer, 97, 589-595.

[29] Segura R., Cierpka C., Rossi M,m Joseph S., Bunjes H., Kähler C.J., (2013), Non-encapsulated thermo-liquid crystals for digital particle tracking thermography/velocimetry in microfluidics, Microfluidics and Nanofluidics, 14( 3-4), 445-456.

[30] Someya S., Li Y., Ishii K., Okamoto K., (2011), Combined twodimensional velocity and temperature measurements of natural convection using a high-speed camera and temperaturesensitive particles, Exp Fluids, 50, 65-73. 
[31] Boiko V.M., Kiselev V.P., Kiselev S.P., Papyrin A.N., Poplavskil S.V., and Fomin V.M., (1996), Interaction of a shock wave with a cloud of particles, Combustion, Explosion, and Shock Waves, 32(2), 191-203.

[32] Fond B., Abram C., Heyes A.L., Kempf A.M., and Beyrau F., (2012), Simultaneous temperature, mixture fraction and velocity imaging in turbulent flows using thermographic phosphor tracer particles, Optics Express, 20(20), 22118-22133.

[33] Abram C., Fond B., Heyes A.L., Beyrau F., (2013), High-speed planar thermometry and velocimetry using thermographic phosphor particles, Applied Physics B, 111(2), 155-160.

[34] Marble F. E., (1970), Dynamics of dusty gases, Ann. Rev. Fluid Mech., 2, 397-446.

[35] Dasgupta S., Jackson R., Sundaresan S., (1998), Gas-particle flow in vertical pipes with high mass loading of particles, Powder Technology, 96, 6-23.

[36] De’ Michieli Vitturi M., Esposti Ongaro T., Neri A., Salvetti M.V., Beux F., (2007), An immersed boundary method for compressible multiphase flows: application to the dynamics of pyroclastic density currents, Comput. Geosci., 11, 183-198.

[37] Raju N. and Meiburg E., (1995), The accumulation and dispersion of heavy particles in forced two-dimensional mixing layers. Part 2: The effect of gravity, Phys. Fluids, 7, 1241-1264.

[38] Pérez-Munuzuri V., (2015), Clustering of inertial particles in compressible chaotic flows, Phys. Rev. E, 91, 052906.

[39] Parmar M., Haselbacher A. and Balachandar S., (2012), Equation of motion for a sphere in non-uniform compressible flows, J. Fluid Mech., 699, 352-375.

[40] Michaelides E. E., (2006), PARTICLES, BUBBLES \& DROPS -Their Motion, Heat and Mass Transfer, World Scientific Publishing Co. Pte. Ltd., Singapore, 2006.

[41] Hirsch C., (2002), Numerical computation of internal and external flows: volume 2: computational methods for inviscid and viscous flows. Chichester, John Wiley \& Sons, 2002.

[42] Laney C.B., (1998), Computational Gas-dynamics, Cambridge University Press.

[43] Anderson J., (1995), Computational fluid dynamics: the basics with applications, Mcgraw Hill, New York, 1995. 


\section{Figure Caption}

Figure 1: particles types : (a) sugar particles, (b) plastic spheres.

Figure 2: (a) digital calibre and (b) precision balance.

Figure 3: Low Subsonic, Hot Flow Facility.

Figure 4: High Subsonic, Cold Flow Facility (a) global view (b) detail of injection zone.

Figure 5: IR camera SC6811.

Figure 6: Thermographic sequence showing the displacement in time of a single particle of sugar for $\xi=1132, V_{J E T}=34 \mathrm{~m} / \mathrm{s}, T_{J E T}=323 \mathrm{~K}, T_{p}=288 \mathrm{~K}$ (sugar particle initial temperature), $\mathrm{Re}_{\mathrm{JET}} \cong$ $1.86 \times 10^{4}, \mathrm{M}=0.094, \mathrm{R}_{\mathrm{p}}=0.845 \mathrm{~mm}, S t \cong 2.47 \times 10^{-3}, F r \cong 6.5 \times 10^{-3}$ (three snapshots at different times, original thermographic picture on the left, digitally enhanced version on the right).

Figure 7: Experimental results (symbols) vs Numerical simulation (solid line) for the same conditions considered in Fig. 6.

Figure 8: Sketch of the different stages of evolution undergone by the particle during its motion from the injector towards the bottom wall.

Figure 9: Nondimensional horizontal distance $\left(l_{x}=\mathrm{L}_{\mathrm{X}} / \mathrm{D}, \mathrm{D}=8 \mathrm{~mm}\right)$ covered by the particle $(\xi=$ 1132, $F r \cong 6.5 \times 10^{-3}$ ) before hitting the bottom wall (located at a nondimensional distance from the jet axis $l_{y}=\mathrm{L}_{\mathrm{y}} / \mathrm{D}=11$ ) as a function of the Stokes number for different values of the jet Mach number (numerical results, the considered range of values of the Stokes number corresponds to the particle radius spanning the dimensional interval $0.845 \leq \mathrm{R}_{\mathrm{p}} \leq 3.38 \mathrm{~mm}$ ).

Figure 10: Snapshot showing the distribution in space of particles for $\xi=1132, V_{J E T}=234 \mathrm{~m} / \mathrm{s}$, $T_{J E T}=288 \mathrm{~K}, T_{p}=288 \mathrm{~K}$ (sugar particle initial temperature), $\mathrm{Re}_{\mathrm{JET}} \cong 1.28 \times 10^{5}, \mathrm{M}=0.65, \mathrm{R}_{\mathrm{p}}=0.845$ $\mathrm{mm}, S t \cong 2.47 \times 10^{-3}, F r \cong 6.5 \times 10^{-3}$ : a) original thermographic picture b) digitally enhanced version.

Figure 11: Numerical simulation of particle swarm dynamics for the same conditions considered in Fig. 9 and different inclinations of the jet: a) $\theta=0.7^{\circ}$, b) $\theta=1^{\circ}$, c) $\theta=1.5^{\circ}$. 
Figure 12: Nondimensional horizontal distance $\left(l_{x}=\mathrm{L}_{\mathrm{x}} / \mathrm{D}, \mathrm{D}=8 \mathrm{~mm}\right)$ covered by the particle before hitting the bottom wall (located at a nondimensional distance from the jet axis $l_{y}=\mathrm{L}_{\mathrm{y}} / \mathrm{D}=11, \mathrm{~L}_{\mathrm{y}}=90$ $\mathrm{mm})$ as a function of the Stokes number for $\xi=1132, F r \cong 6.5 \times 10^{-3}, \mathrm{M}=0.65$ and different values of the jet inclination angle (numerical results).

Figure 13: Thermographic sequence showing the displacement in time of a single particle of plastic for $\xi=1078, V_{J E T}=308 \mathrm{~m} / \mathrm{s}, T_{J E T}=288 \mathrm{~K}, T_{p}=288 \mathrm{~K}$ (plastic particle initial temperature), $\operatorname{Re}_{\mathrm{JET}} \cong$ $4.22 \times 10^{5}, \mathrm{M}=0.9, \mathrm{R}_{\mathrm{p}}=3 \mathrm{~mm}, \mathrm{St} \cong 5 \times 10^{-3}, \mathrm{Fr} \cong 1.65 \times 10^{-3}$ (three snapshots at different times, original thermographic picture on the left, digitally enhanced version on the right).

Figure 14: Thermographic Sequence (digitally enhanced pictures) for the same conditions considered in Fig. 13.

Figure 15: Experimental particle trajectory for the same conditions considered in Fig. 13.

Figure 16: Nondimensional horizontal distance $\left(l_{x}=\mathrm{L}_{\mathrm{X}} / \mathrm{D}, \mathrm{D}=20 \mathrm{~mm}\right)$ covered by the particle before hitting the bottom wall (located at a nondimensional distance from the jet axis $l_{y}=\mathrm{L}_{\mathrm{y}} / \mathrm{D}=4.5$, $\mathrm{L}_{\mathrm{y}}=90 \mathrm{~mm}$ ) as a function of the Stokes number for $\xi=1078, \mathrm{Fr} \cong 1.65 \times 10^{-3}, \mathrm{M}=0.9$ and different values of the jet inclination angle. 